\title{
Isogeometric analysis for strain field measurements
}

\author{
Thomas Elguedj ${ }^{* a}$, Julien Réthoréa ${ }^{\mathrm{a}}$, Aurélien Buteri ${ }^{\mathrm{b}}$ \\ ${ }^{a}$ Université de Lyon, CNRS \\ INSA-Lyon, LaMCoS UMR5259, F-69621, Villeurbanne, France \\ ${ }^{b}$ Université de Lyon, CNRS \\ INSA-Lyon, MATEIS UMR5510, F-69621, Villeurbanne, France
}

\section{Abstract}

In this paper, the potential of Isogeometric Analysis for strain field measurement by Digital Image Correlation is investigated. Digital Image Correlation (DIC) is a full field kinematics measurement technique based on gray level conservation principle and the formulation we adopt allows for using arbitrary displacement bases. The high continuity properties of Non-Uniform Rational B-Spline (NURBS) functions are exploited herein as an additional regularization of the initial ill-posed problem. $k$-refinement is analyzed on an artificial test case where the proposed methodology is shown to outperform usual finite element based DIC. Finally a fatigue tensile test on a thin aluminum sheet is analyzed. Strain localization occurs after a certain number of cycles and combination of NURBS into a DIC algorithm clearly shows a great potential to improve the robustness of non-linear constitutive law identification.

Key words: digital image correlation, NURBS, strain field measurement, isogeometric analysis

\section{Introduction}

Since a decade, full field measurement techniques have initiated a kind of revolution in solid mechanics. They offer the capabilities to access the displacement or strain not only at some pre-defined gauge location but on the whole surface of the sample (for conventional 2D techniques). Digital Image Correlation (DIC) (Sutton et al. [1]) is one of the most appealing of these full field measurement techniques as it is really user friendly: one only needs to take a series of digital images of the sample. Depending on the observation scale, the rough surface of the studied specimen can be used as a natural texture or a painted random pattern can be spraid on the sample. The randomness of the texture is then exploited to solve the optical flow conservation principle, which finally gives access to the displacement at each pixel of the reference image.

For non-linear constitutive behavior identification, full field measurements have become unavoidable because they necessitate to exploit experimental tests up to strain localization, failure or necking. In

*Corresponding author: Thomas Elguedj, Lamcos MSE, bâtiment Jacquard, 27 avenue Jean Capelle, 69621 Villeurbanne Cedex, France.

Email addresses: thomas.elguedj@insa-lyon.fr (Thomas Elguedj), julien.rethore@insa-lyon.fr (Julien Réthoré), aurelien.buteri@insa-lyon.fr (Aurélien Buteri) 
this context, the natural measured field (the displacement field) must be converted into strains to feed identification algorithms. As a derivative of an experimental quantity, strain fields often exhibit large noise levels that must be accounted for in the identification procedure (see, e.g., Avril et al. [2]). Another route, the one we follow herein, is to work on the measurement step itself. The aim of this paper is to present a DIC strategy that allows for strain measurements with higher resolution and lower noise levels. Starting from piecewise linear function, one can increase the degree to increase the resolution. However, due to the illposedness of the optical flow principle, this will also increase noise measurement. The remedy is to increase the "amount" of regularization. Increasing the continuity of the approximation functions of the displacement plays this role. We thus look for functions that can be " $k$ " enriched, that is with higher polynomial degree while high continuity is maintained, without a prohibitive increase of the number of degrees of freedom. Non-Uniform Rational B-Spline functions (NURBS) have such capacities.

NURBS functions are standards in Computer Aided Design, computer graphics and animation. These functions grew out of the pioneering work of Pierre Bézier's development of Bézier curves and surfaces in the late 1960s. B-Spline and later NURBS and T-Splines were developed since then (see, e.g., Farin [3], Piegl and Tiller [4], Cohen et al. [5], Rogers [6]). Despite a strong coupling between design and analysis in the global design of industrial products, such functions were not used in the analysis until recently. The work of Hughes et al. [7], Cottrell et al. [8] on Isogeometric Analysis introduced NURBS functions in a "finite element" framework to represent both geometry and solution fields. Despite a real interest in the geometric representation built into isogeometric analysis, the smoothness of NURBS and B-Spline functions is the key ingredient that drove the use of the approach in various domains of analysis such as turbulence and fluidstructure interactions (Bazilevs et al. [9, 10], Akkerman et al. [11])); Cahn-Hilliard phase field modeling (Gomez et al. [12]), incompressible problems (Auricchio et al. [13], Elguedj et al. [14])) and structural dynamics (Hughes et al. [15]). We propose in this paper to couple isogeometric analysis and DIC in a similar way as proposed by Besnard et al. [16] with piecewise linear finite elements.

The paper is organized as follows: section 2 is dedicated to the optical flow conservation principle, section 3 presents the construction of NURBS functions, an example with artificially deformed images is then proposed in section 4 while an experiment on a thin aluminum sheet is analyzed in section 5 . In section 6 we draw conclusions.

\section{Digital Image Correlation algorithm}

\subsection{Optical flow conservation principle}

If successive images (mathematically analyzed as continuous gray level functions of the pixel co-ordinates in the image frame) of the same sample are captured during its motion, a displacement $\mathbf{u}$ produces an advection of the local texture of the images. When analyzing a pair of images $f$ and $g$, the optical flow 
conservation reads

$$
f(\mathbf{x})=g(\mathbf{x}+\mathbf{u}(\mathbf{x})) .
$$

71 with

72 and

The principle of DIC is to determine $\mathbf{u}$ as accurately as possible. The problem is illed-posed by nature as a vector field is searched for from a scalar equation. Further, the displacement may be measurable along the direction of the gradient of $g$ only. Thus, the first step is to try and solve it in an "average" sense, i.e., by minimizing a global error

$$
\eta^{2}=\iint_{\Omega} \Phi^{2}(\mathbf{x}) \mathrm{d} \mathbf{x}
$$

where $\Omega$ is the domain of interest and $\Phi(\mathbf{x})$ defines the local correlation error:

$$
\Phi(\mathbf{x})=[f(\mathbf{x})-g(\mathbf{x}+\mathbf{u}(\mathbf{x}))]
$$

The usual approach consists in maximizing a correlation coefficient (Sutton et al. [1, 17, 18]) by searching for a piecewise constant, bilinear or other canonical form of displacement field. The image is thus subdivided in zones of interest that can overlap and the maximization is performed independently over each of these zones. Note that B-spline functions have already been used by Cheng et al. [19] in this context.

It has been shown in Besnard et al. [16] that prescribing displacement continuity over the entire domain of interest plays a regularizing role. Because of the ill-posedness of the problem, the "amount" of regularization is directly related to noise sensitivity and thus measurement error and uncertainty.

\subsection{Resolution}

We use in the following the global approach proposed in Besnard et al. [16]. After a description basis is adopted for the displacement field

$$
\mathbf{u}(\mathbf{x})=\sum_{n \in \mathcal{N}} a_{n} \boldsymbol{\psi}_{n}(\mathbf{x})=[\mathbf{\Psi}(\mathbf{x})]\{\mathbf{U}\}
$$

an iterative Newton procedure is initiated in order to minimize $\eta^{2}$ (Eq. (2)). A sequence of linear system is solved until convergence of the solution increment $\mathrm{d} \mathbf{U}$ is obtained:

$$
[\mathbf{M}]^{(i)}\{\mathrm{d} \mathbf{U}\}=\{\mathbf{b}\}^{(i)}
$$

$$
M_{n m}^{(i)}=\iint_{\Omega}\left(\boldsymbol{\psi}_{n} \cdot \nabla g\left(\mathbf{x}+[\mathbf{\Psi}]\{\mathbf{U}\}^{(i)}\right)\right)\left(\boldsymbol{\psi}_{m} \cdot \nabla g\left(\mathbf{x}+[\mathbf{\Psi}]\{\mathbf{U}\}^{(i)}\right)\right) \mathrm{d} \mathbf{x},
$$

$$
b_{n}^{(i)}=\iint_{\Omega}\left(\boldsymbol{\psi}_{n} \cdot \nabla g\left(\mathbf{x}-[\mathbf{\Psi}]\{\mathbf{U}\}^{(i)}\right)\right)\left(f(\mathbf{x})-g\left(\mathbf{x}+[\mathbf{\Psi}]\{\mathbf{U}\}^{(i)}\right)\right) \mathrm{d} \mathbf{x},
$$

$\nabla$ denoting spatial derivation and $i$ the current iteration number. 


\subsection{Numerical aspects}

To reduce numerical costs, the tangent operator $[\mathbf{M}]^{(i)}$ is not computed at each iteration. In practice, the tangent operator is set to the final one, i.e., replacing $\nabla g\left(\mathbf{x}+[\mathbf{\Psi}]\{\mathbf{U}\}^{(i)}\right)$ by $\nabla f(\mathbf{x})$ which can be computed once for all. For large deformations or when the choice of a simple initial solution is not the best choice, one can adopt a balanced tangent operator by weighting the contribution of the current search direction (using $\left.\nabla g\left(\mathbf{x}+[\mathbf{\Psi}]\{\mathbf{U}\}^{(i)}\right)\right)$ and the final one (using $\nabla f(\mathbf{x})$ ).

The derivation operations are performed numerically by finite differences on the pixel grid of the image. The integrals in $M_{n m}^{(i)}$ and $b_{n}^{(i)}$ are computed as discrete sums over the set of pixels in the domain of interest, these pixels are considered as integration cells with one Gauss point per cell.

The sub-pixel interpolation of the deformed image (the values of $g$ at non-integer value of $\mathbf{x}+[\mathbf{\Psi}]\{\mathbf{U}\}^{(i)}$ ) is performed using a spline-cubic interpolation of the gray level from the neighbor pixels, see Bornert et al. [20] for an overview.

\subsection{Multigrid algorithm}

As mentionned in Besnard et al. [16], a multigrid resolution is an essential feature of the global correlation algorithm we use herein. Indeed, due to the ill-posed nature of the problem and the influence of noise, a strategy must be adopted in order to introduce progressively the shortest wave lengths of the solution and to avoid local minima. This has been decribed and studied in details in Réthoré et al. [21].

The idea is to start with a coarsened description not only of the displacement field but also of the images $f$ and $g$ and then to introduce step by step the whole frequency content of the images and displacement basis. For these purposes, we define a "grain" as a generic denomination of a pixel whatever the scale. At scale $n+1$, a grain contains $2 \times 2$ grains of scale $n$, at scale 1 a grain is a pixel. Then the value of $f$ or $g$ in gray level for scale $n+1$ at each grain is set to the mean value of the gray level of its "sub"-grains at scale $n$. This procedure is illustrated by Figure ?? and can be seen as a restriction operation $\mathrm{R}$ that reads:

$$
f^{n+1}\left(\mathbf{x}_{G r}\right)=\mathrm{R}\left(f^{n}, \mathbf{x}_{G r}\right)=\frac{1}{2^{2}} \sum_{g r \in G r} f^{n}\left(\mathbf{x}_{g r}\right)
$$

where $\mathbf{x}_{G r}$ denotes the position of grain $G r$ of scale $n+1$ in the reference image $f^{n+1}$ at this scale and $\mathbf{x}_{g r}$ the position of grain $g r$ of scale $n$ in the reference image $f^{n}$. The short wave lengths of the image texture are thus filtered progressively. For the deformed image $g$, its correction $g\left(\mathbf{x}+[\mathbf{\Psi}]\{\mathbf{U}\}^{(i)}\right)$ is first performed and then the coarse-graining step is performed.

The displacement discretization is finite element based and regular meshes are used. It is thus easy to coarsen the description of the unknown field. At scale $n$, elements are $h \times h$ grains and thus at scale $n+1$ they are still $h \times h$ grains since grains have been coarsened. The prolongation of the displacement is 
performed using the bilinear interpolation of the finite element shape functions (see also Figure ??)

$$
U_{n, p}=\mathrm{P}\left(U_{n+1}, \mathbf{x}_{p}\right)=\sum_{j \in \mathcal{N}_{e}^{n+1}} U_{n+1, j} N_{j}^{n+1}\left(\mathbf{x}_{n}\right),
$$

where $\mathrm{P}$ is the prolongation operator, $U_{n, p}$ is the displacement at node $p$ of level $n,\left(\mathbf{x}_{p}\right)$ its position, $N_{j}^{n+1}$ the finite element shape functions supported by nodes $j$ in the set of all nodes $\mathcal{N}_{e}^{n+1}$ at scale $n+1$, and $U_{n+1, j}$ the nodal values of the displacement at level $n+1$.

Note that for all the scales but the finest one, first order polynomial shape functions are used whereas for the finest scale NURBS functions will be used in the sequel. For the transition between scale 2 and scale 1 , after a linear interpolation of the nodal displacement from the coarse to the fine mesh using $\mathrm{P}$, the initial displacement on a $p$-degree basis (see section 3 ), is obtained by $L^{2}$ projection. In practice 3 to 5 scales are used. The first computation on the coarsest one is initialized by estimating a rigid body translation at each node of the corresponding mesh. One could think of using NURBS functions at every scale of the multigrid algorithm in the present case. This would render the algorithm more complex, especially in order to form prolongation and restriction operators and does not improve the results further. Indeed, the only role of the resolution of the coarsened problems is to avoid local minima, the final resolution being done on the fine grid, we only need the performance of NURBS functions at that particular scale.

\section{Non-Uniform Rational B-Splines and Isogeometric Analysis}

Non-Uniform Rational B-Splines (NURBS) are a standard tool for describing and modeling curves and surfaces in computer aided design and computer graphics (see Piegl and Tiller [4], Rogers [6] for an extensive description of these functions and their properties). In this work, we use NURBS as a DIC/analysis tool, which is referred to as isogeometric analysis by Hughes et al. [7], Cottrell et al. [8]. The aim of this section is to present a brief overview of features and properties of NURBS-based isogeometric analysis for 2D DIC problems.

\subsection{B-spline and NURBS functions}

B-splines are piecewise polynomial functions with a prescribed degree of continuity. Univariate Bspline basis functions are constructed from a knot vector, a set of coordinates in parametric space, $\Xi=$ $\left\{\xi_{1}, \xi_{2}, \ldots, \xi_{n+p+1}\right\}$, where $\xi_{i} \in \mathbb{R}$ is the $i^{\text {th }}$ knot, $i$ is the knot index, $i=1,2, \ldots, n+p+1, p$ is the polynomial order, and $n$ is the number of basis functions. More than one knot can be placed at the same location in the parametric space. If $m$ is the multiplicity of a given knot, the functions are $C^{p-m}$ continuous at that location. If the knots are equally spaced, the knot vector is said to be uniform and non-uniform otherwise. A knot vector is referred to as open if its first and last knots have multiplicity $p+1$. This results in the 
basis being interpolatory at the endpoints of the interval. A knot vector is referred to as periodic if its first and last knots have unit multiplicity.

B-spline basis functions for a given order $p$, are defined recursively in the parametric space by way of the knot vector $\Xi$. Beginning with piecewise constants $(p=0)$ we have

$$
N_{i, 0}(\xi)= \begin{cases}1 & \text { if } \xi_{i} \leq \xi<\xi_{i+1} \\ 0 & \text { otherwise }\end{cases}
$$

For $p=1,2,3, \ldots$, the basis is defined by the Cox-de Boor recursion formula:

$$
N_{i, p}(\xi)=\frac{\xi-\xi_{i}}{\xi_{i+p}-\xi_{i}} N_{i, p-1}(\xi)+\frac{\xi_{i+p+1}-\xi}{\xi_{i+p+1}-\xi_{i+1}} N_{i+1, p-1}(\xi)
$$

Let $d$ denote the number of spatial dimensions. A B-spline curve in $\mathbb{R}^{d}$ is defined as follows:

$$
\mathbf{C}(\xi)=\sum_{i=1}^{n} N_{i, p}(\xi) \mathbf{B}_{i}
$$

where $\mathbf{B}_{i} \in \mathbb{R}^{d}$ denotes control point $i$.

Important properties of B-spline curves are:

- Affine Covariance: An affine transformation of a B-spline curve is obtained by applying the transformation to its control points.

- Convex Hull: A B-spline curve lies within the convex hull of its control points (see Rogers [6] for the relationship between the convex hull and the polynomial degree of the curve).

- Variation Diminishing: A B-spline curve in $\mathbb{R}^{d}$ cannot cross an affine hyperplane of codimension 1 (e.g., , a line in $\mathbb{R}^{2}$, a plane in $\mathbb{R}^{3}$ ) more times than does its control polygon (see Piegl and Tiller [4]).

With the use of tensor products, the concept can be extended to multiple dimensions. For a B-spline surface, we start by defining two knot vectors $\Xi=\left\{\xi_{1}, \xi_{2}, \ldots, \xi_{n+p+1}\right\}$ and $\mathcal{H}=\left\{\eta_{1}, \eta_{2}, \ldots, \eta_{m+q+1}\right\}$ and an $n \times m=\mathbf{s}$ net of control points $\mathbf{B}_{i, j}=\mathcal{B}_{\mathbf{k}}$. One-dimensional basis functions $N_{i, p}$ and $M_{j, q}$, with $[i, j] \in[\{1, \ldots, n\},\{1, \ldots, m\}]$, of order $p$ and $q$ respectively are defined from the knot vectors, and their tensor product forms the two-dimensional basis function $\mathcal{N}_{\mathbf{k}, \mathbf{r}}(\boldsymbol{\zeta})=N_{i, p}(\xi) M_{j, q}(\eta)$, where $\boldsymbol{\zeta}=\xi \times \eta, \mathbf{k}=i \times j$ and $\mathbf{r}=p \times q$. The B-spline surface is defined by the extension of Eq. (12):

$$
\mathcal{S}(\zeta)=\sum_{\mathbf{k}=1}^{\mathbf{s}} \mathcal{N}_{\mathbf{k}, \mathbf{r}}(\zeta) \mathcal{B}_{\mathbf{k}}
$$

A similar description can be constructed for higher dimensional spaces, for example to form a B-spline volume in $3 \mathrm{D}$.

A rational B-spline object in $\mathbb{R}^{d}$ is obtained from the projection of a nonrational (polynomial) B-spline object in $\mathbb{R}^{d+1}$. To obtain a NURBS curve in $\mathbb{R}^{2}$, we start by defining a set of control points called 
projective points $\mathbf{B}_{i}^{w} \in \mathbb{R}^{3}$, associated with a B-spline curve in $\mathbb{R}^{3}$ with knot vector $\Xi$. The control points for the NURBS curve are given by

$$
\left(\mathbf{B}_{i}\right)_{j}=\frac{\left(\mathbf{B}_{i}^{w}\right)_{j}}{\omega_{i}} \text { with } j=1,2,
$$

where $\left(\mathbf{B}_{i}\right)_{j}$ is the j-th component of $\mathbf{B}_{i}$ and $\omega_{i}=\left(\mathbf{B}_{i}^{w}\right)_{3}$ is the third component of $\mathbf{B}_{i}^{w}$ and is referred to as the i-th weight. The NURBS basis function of order $p$ are defined by the following equation:

$$
R_{i, p}(\xi)=\frac{N_{i, p}(\xi) \omega_{i}}{\sum_{i=1}^{n} N_{i, p}(\xi) \omega_{i}} .
$$

The NURBS curve is then defined by

$$
\mathbf{C}_{n}(\xi)=\sum_{i=1}^{n} R_{i, p}(\xi) \mathbf{B}_{i} .
$$

This can be generalized to define NURBS surfaces and volumes.

\subsection{Isogeometric analysis for DIC}

The main features of NURBS based isogeometric analysis are given in Cottrell et al. [8] and references herein. The reader can find many of the details and some applications in Hughes et al. [7], Cottrell et al. [8], Bazilevs et al. [9, 10], Akkerman et al. [11], Gomez et al. [12], Auricchio et al. [13], Elguedj et al. [14], Hughes et al. [15].

Some features of the NURBS based isogeometric analysis that are build into the method in order to perform mechanical computations are not necessary when applied to DIC. Based on the observation that the computational domain is a regular rectangular grid formed on the pixel discretization of the image, and the fact that the geometric description of the area of interest is not needed, the physical and parametric meshes are superposed (see Figure ??). Another interesting point is that no boundary conditions need to be applied on the domain. Consequently having the basis function to be interpolatory on the boundary of the domain is not necessary. This means that the use of open knot vectors is not mandatory and other types of knot vectors can be considered We propose here to use periodic B-spline functions in a similar way as proposed in Bazilevs et al. [9] for periodic boundary conditions imposition. This necessitates to introduce additional knots in the vector in order to produce incomplete basis functions on the first and last elements. This has to be done in order to preserve the partition of unity property of the basis. The use of periodic knot vectors produces the same number of basis functions as with open knot vectors, thus the same number of unknowns. However, it is computationally more efficient as we only need to form the 1D basis functions and integral contributions based on one element, and reuse this evaluation on all the elements in all the spatial directions. This is particularly true as we employ a pixel based numerical integration which consists in a sub-quadrilateral decomposition of elements with a one Gauss point rule in each pixel. A comparison of basis functions generated from open and modified periodic knot vectors is shown in Figure ?? for quadratic 
to quintic B-spline basis. We can observe that with open knot vectors, the basis functions in boundary elements have a different shape than the ones in the interior elements. On the contrary, with periodic knot vector, basis functions have the same shape in all the elements, which allow us to compute them once and for all for one 1D element and reuse these values for all the mesh

The choice of using periodic knot vectors was done only to simplify numerical implementation and improve the numerical cost of the method. The example presented in the sequel were also performed using open knot vectors and no difference was observed in the result.

It is interesting to note here that the basis functions obtained for $\mathcal{C}^{0}$ linear NURBS are exactly the same as the standard piecewise linear finite elements, therefore for that particular case, the method is exactly the same as the one introduced in Besnard et al. [16].

\section{Artificial test}

The first example we present here can be viewed as an a priori performance analysis of the method. A common question in DIC as in analysis is the choice of the element size. In DIC, this point is a matter of compromise between correlation and interpolation errors. The correlation error arises from the ill-posed nature of the problem, and the interpolation error when the actual displacement cannot be interpolated with the chosen basis functions. It is important at this point to estimate the contribution of these two errors, and to see how high order NURBS functions behave when compared to standard low order finite element functions. Another interesting point comes from the fact that DIC is not a purely numerical problem but is affected by experimental uncertainty. Thus, the sensitivity to measurement noise also needs to be addressed.

To study the correlation error, we employ a common technique which consists in performing a DIC analysis over the domain of interest of the initial image with a deformed image which is artificially translated by $u_{t}$ pixels in the horizontal direction. As the prescribed displacement is constant, no interpolation error arises at this step. For the interpolation error, we choose a displacement field $u_{s}$ that is not in the interpolation space of the basis functions, and compare it with its $L^{2}$ projection onto the basis functions. As no DIC problem is solved, no correlation error arises at this step. Finally, we solve a complete DIC problem based on the same predefined solution $u_{s}$. We impose the reference displacement field $u_{s}$ on a given picture to create a simulated deformed picture. This is similar to the use of problems with a known analytical solution in numerical methods for convergence analysis and is a common strategy employed in the DIC literature to study algorithm efficiency and "convergence".

As proposed in Bornert et al. [20], we only impose a displacement field with a horizontal component. We choose the following displacement form:

$$
u_{s}(X)=U_{0} \sin \left[\frac{\omega_{x}}{2 \pi}\left(1-\left|2 \frac{X}{\max (X)}-1\right|\right)^{2}\right],
$$


where $\omega_{x}=5 \pi^{2}, U_{0}=1$ pixel and $X$ is the horizontal coordinate in the image reference axis. This produces a symmetric sinusoidal displacement field with an increasing frequency from the boundary of the image to the center. The corresponding component of the strain field $E_{x x}$ also presents a sinusoidal shape with increasing frequency and amplitude from the boundary to the center of the image. We only impose a horizontal displacement as it allows us to study the variation of the measurement error in the vertical direction. Contrary to standard numerical simulation with a similar solution, we expect the computed horizontal displacement component to vary along different vertical lines.

\subsection{A priori performance and noise sensitivity analysis}

Following Réthoré et al. [22], we can study noise sensitivity by doing a perturbation analysis of Eq. (5). The idea is to consider that both the reference and deformed image are corrupted by a random noise. This noise is supposed to be of zero mean and spatially uncorrelated. For simplicity, we consider that the deformed image is noiseless and that the deviation from optical flow conservation given in Eq. (1) allows us to define an effective noise that only affects the reference image. Consequently, this effective noise has twice the variance of the initial noise, that is $2 \sigma^{2}$. The perturbation induced on the "displacement" vector [dU] is of zero mean but spatially correlated. Its correlation kernel $[\mathbf{C}]$ is given by

$$
<\{\mathrm{d} \mathbf{U}\}\{\mathrm{d} \mathbf{U}\}>=2[\mathbf{C}] \sigma^{2}=2[\mathbf{M}]^{-1} \sigma^{2}
$$

Figure ?? shows the square root of the diagonal elements of $[\mathbf{C}]$ averaged over all the degrees of freedom. The curves are plotted with the contribution of boundary degrees of freedom for $\mathcal{C}^{p-1}$ in Figure ?? and $\mathcal{C}^{0}$ NURBS in Figure ??, and without the contribution of the boundary dofs in Figure ?? and Figure ??. We can see that the noise sensitivity decreases as the element size increases for all values of the degree for $\mathcal{C}^{0}$ NURBS and for degrees up to $p=3$ for $\mathcal{C}^{p-1}$ NURBS with boundary dofs. We can also see in both cases that the sensitivity increases with the degree of the NURBS functions, no matter the continuity. Further, for $\mathcal{C}^{p-1}$ NURBS with boundary dofs, the slope of the sensitivity vs element size is changing as the degree is increased and as the element size is increasing, in particular, the sensitivity is constant then increases for the quartic case. Without boundary dofs, the results are almost identical for $\mathcal{C}^{0}$ NURBS that with boundary dofs. On the contrary, with $\mathcal{C}^{p-1}$ NURBS, we can see that the sensitivity decreases with the element size for all degrees and with lower error levels than with $\mathcal{C}^{0}$ NURBS. This indicates that higher continuity provides less sensitivity to noise for interior dofs.

We also plot in Figure ?? sensitivity maps as the norm of the rows of $[\mathbf{C}]$, these maps are plotted with a value for each degree of freedom and are not interpolated using basis functions as for displacement fields. On Figure ??, we plot these maps for $\mathcal{C}^{p-1}$ NURBS. These sensitivity maps are not homogeneous due to the heterogeneous nature of the gradient of the reference image. We can clearly see that the boundary degrees of freedom have a sensitivity that is much higher than on the inside of the domain. The support of these 
degrees of freedom is much smaller, one half and one fourth on the corners, than the one of the inside degrees of freedom. We can see that the difference between the sensitivity on the boundary and on the inside is increasing with the degree of the functions. We also plot on Figure ?? the same maps only with the degrees of freedom that have a zero contribution on the boundary of the domain. We can see that the maps look similar and that the scale is more or less multiplied by a factor of five each time the degree is increased. Finally we plot on Figures ?? and ?? the same maps for $\mathcal{C}^{0}$ NURBS for $p=1,2,3$ for all the degrees of freedom and for the interior degrees of freedom.

\subsection{Error analysis}

The error analysis is decomposed into three steps in order to analyze the correlation and interpolation errors separately and together. The first step consists in studying the correlation error by analyzing an image deformed by a rigid body translation. The second step is devoted to the analysis of the interpolation error. Finally the last step consists in analyzing the results of the DIC algorithm using an image deformed by the same displacement field as for the step 2; this analysis produces what is called the total error.

For the first step, i.e., the analysis of the correlation error, the value of $u_{t}=0.5$ pixel is chosen because it produces the maximum correlation error. The displacement prescribed at steps 2 and 3 is the one given in Eq. (17). For step 2, the $L^{2}$ projection is performed in the following way:

$$
\operatorname{Arg}\left[\min \iint_{\Omega}\left(u-u_{s}\right)^{2} \mathrm{~d} \mathbf{x}\right] .
$$

For each step, the error is obtained by taking the standard deviation of the gap between the computed displacement (by DIC or $L^{2}$ projection) and the prescribed displacement, i.e., the RMS (root mean square) error.

By comparing the results of the last step with the first ones, we are able to evaluate the competition between the correlation and the interpolation errors in DIC analysis with given parameters. We performed such an analysis with varying values for the element size $h$, the polynomial degree $p$, and the continuity $i . e .$, $C^{0}$ or $C^{p-1}$. This allows us to estimate the influence of these parameters on the performance of NURBS based DIC. In all the results presented below, the strain field is computed as in the usual finite elements setting, that is by computing the derivatives of the basis functions at the integration points.

The results are presented in Figures ?? to ??. In Figure ??, we plot the correlation error and the interpolation error for displacement and strain as a function of the element size for $\mathcal{C}^{0}$ and $\mathcal{C}^{p-1}$ NURBS for $p=1,2,3$. We can see that for both fields, the correlation error decreases as a power law of $h$ whereas the interpolation error increases as a power law of $h$. The slope for the correlation error is about 1 for all cases for the displacement and about 2 for the strain. For $\mathcal{C}^{p-1}$ NURBS the error is almost independent of the degree, whereas for $\mathcal{C}^{0}$ NURBS the error level increases with the degree with a relatively constant slope. The error level is approximately increased by a factor of 10 when the degree is increased by one unit. For 
the interpolation error, as it could be anticipated, the slopes are respectively of $2,3,4$ for the displacement and $1,2,3$ for the strain with $p=1,2,3$. The error levels are this time in favor of the $\mathcal{C}^{0}$ functions compared to the $\mathcal{C}^{p-1}$ functions for both fields. We can also note that the slopes tend to diminish as $h$ becomes small for the $\mathcal{C}^{0}$ functions.

In Figure ??, we plot the RMS error on the horizontal displacement on the top and on the strain on the bottom as a function of the element size in pixels. We plot on the same graphs the correlation error with plain lines, the interpolation error with dashed lines and the total error with square marks. On the left part, we use $\mathcal{C}^{0}$ piecewise linear functions, in the middle we use $\mathcal{C}^{0}$ piecewise cubic NURBS and on the right, we use $\mathcal{C}^{2}$ piecewise cubic NURBS. Note that for $\mathcal{C}^{0}$ piecewise cubic NURBS the algorithm did not converge for 8-pixels elements. With $\mathcal{C}^{0}$ piecewise linear functions, the total error is governed by the interpolation error and lowest error levels are obtained for 8-pixels elements. On the contrary, for the $\mathcal{C}^{0}$ cubic NURBS, the total error is mostly governed by the correlation error for both the displacement and the strain. The lowest error is attained for 32-pixels elements. For $\mathcal{C}^{p-1}$ NURBS functions, the competition between correlation and interpolation error is well balanced and an optimal choice of the element size for this particular case appears to be 16 pixels with $\mathcal{C}^{2}$ cubic functions. We might also want to consider the intersection point between the correlation and interpolation error curves. This point defines the minimum total error that is potentially reachable with the basis functions considered. With $\mathcal{C}^{p-1}$ NURBS, we can see that for both the displacement and the strain, this "optimal" point gives smaller errors when compared to $\mathcal{C}^{0}$ NURBS.

The horizontal displacement and strain solution profiles can be seen in Figures ?? to ??. We plot the imposed and computed fields versus the horizontal coordinate in pixels. We superpose the values of the computed fields for all vertical coordinates; this allows us to see the variation of the solution along the vertical direction. We plot the results with $\mathcal{C}^{p-1}$ and $\mathcal{C}^{0}$ functions for $8,16,32$ and 64 pixels per elements. In Figure ??, the missing figures correspond to cases for which the method did not converge no matter the number of scales used in the multigrid algorithm and the initial value of the displacement considered. We can clearly see on the strain curves that when the element size starts to increase, the solution is much better: it has fewer oscillations in the horizontal direction and fewer variations along the vertical direction. Then when the element size keeps increasing, the discretization error dominates and we are not able to capture the solution. The comparison of the $\mathcal{C}^{p-1}$ and $\mathcal{C}^{0}$ cases clearly shows the superiority of smooth high order functions for strain measurements (Figures ?? and ??).

Finally, we plot in Figure ?? a three-dimensional visualisation of the $\varepsilon_{x x}$ strain component (amplified 1000 times in the $z$-direction) and of the error on the $\varepsilon_{x x}$ strain component (amplified 3000 times in the $z$-direction). We plot the results obtained with 16 pixels per elements with $\mathcal{C}^{0}$ linear, $\mathcal{C}^{2}$ cubic and $\mathcal{C}^{0}$ cubic NURBS. This figure also shows that we obtain much better results in terms of strain with higher order and higher continuity of the discretization functions. 


\section{Experimental test}

This section is dedicated to an experimental test performed on a thin aluminum sheet.

\subsection{Material and devices}

The present tests have been carried out on co-rolled aluminum alloys thin-sheet currently used in thermal exchangers for motor industry. This kind of multi-material structure is made out of two or three different aluminum alloys having different liquidus temperatures $\left(T_{\text {clad-liquidus }}<T_{\text {core-liquidus }}\right)$. A specific temperature increase leads to the melting of the clad and, by capillarity, to the formation of brazed joints between beforehand-assembled exchanger components. The core material ensures mechanical strength and thermal properties whereas clads ensure mechanical exchangers cohesion after the joining process. The low thickness of the structure (between $0.2-0.3 \mathrm{~mm}$ before brazing) allows to optimize the thermal exchange capacity thanks to the increase of the exchange surface area. The material configuration studied is made up of $\mathrm{Al}-\mathrm{Mg}$ alloy for the core material (75\% of the thickness) and Al-Si and Al-Zn alloys (respectively $15 \%$ and $10 \%$ of the thickness) for the two clads. Samples were manufactured from industrial co-rolled sheets with a theoretical section of $15 \times 0,27 \mathrm{~mm}^{2}$. Other sample dimensions are shown in Figure ??(a). Observations were carried out on Al-Si clad before the brazing step. Fatigue tests have been performed at room temperature at MATEIS lab using an 8516 INSTRON hydraulic testing device combined with a $5 k N$ loadcell (maximum stress of $180 \mathrm{MPa}$, load ratio of $R=0.1$ and sinusoidal waveform at $F=10 \mathrm{~Hz}$ ). The thickness of each samples have been controlled with a thickness indicator Mitutoyo IP65.

The images are $1200 \times 1600$ pixels with 8-bit digitization. The reference image is shown in Figure ??(b). This Figure also presents the boundary of the domain of interest and the mesh for 16-pixels elements. Figure ??(c) and (d) show the deformed images at 6000 and 8500 cycles. The pixel size for these images is pix $2 \mathrm{~m}=10.7 \mu \mathrm{m}$. The part of the sample shown in the image corresponds to a zoom on the active region at the center of the specimen. Note that the images are actually rotated of $90^{\circ}$ compared to the representation of Figure ??(a).

\subsection{Results}

First, the analysis is carried out using 16-pixel elements with $\mathcal{C}^{0}$ piecewise linear functions. Figure ?? shows the displacement results at 8500 cycles: the map ?? gives the horizontal displacement in pixel, the map ?? the vertical displacement and the deformed mesh is shown on Figure ??. Figure ?? shows the local correlation residual $(|f(\mathbf{x})-g(\mathbf{x}+\mathbf{u}(\mathbf{x}))|)$ in gray level in \% of the dynamic of the reference image (223 gray levels). Except inside the zone where strain localization is suspected, the correlation error is lower than $2 \%$. Inside the localization zone, maximum values of about $8 \%$ are obtained. This allows a high level of confidence in the results. From Figure ??, it appears that the deformation is localized within a band which orientation with respect to the loading direction is about $65^{\circ}$. For a deeper analysis, the three components 
of the Green-Lagrange strain tensor $\left(E_{x x}, E_{y y}\right.$ and $\left.E_{x y}\right)$ are presented in Figure ?? after 8500 cycles. It is confirmed that the strain is localized within a band whose width is about 200 pixels with an average level of $E_{x x}$ of about $15 \%$. It is also observed that conjugate bands (with a $-65^{\circ}$ angle with respect to the loading direction) have developed but with lower strain levels (about 5\%). Figure ?? reveals that $E_{y y}$ is localized along the same patterns as $E_{x x}$ with a strain intensity of about 2 to $3 \%$. Figure ?? concerns $E_{x y}$ which is almost homogeneous and about noise measurement.

Figure ?? shows $E_{x x}$ after 8500 cycles for different discretization bases for the displacement. For a $\mathcal{C}^{0}$ linear basis, Figures ?? to ?? show the results for 16, 32 and 64-pixel elements. It is observed that the noise is reduced when the element size increases; but a coarser description of the localization patterns is obtained. For comparison, the same analysis is performed using a $\mathcal{C}^{2}$ cubic NURBS functions in Figures ?? to ??. The same observations arise concerning the effect of noise measurement, but the higher continuity of $\mathcal{C}^{2}$ cubic NURBS functions allows for a better description of the strain localization for a given mesh size. Concerning correlation error, average values of $1.43 \%, 1.47 \%$ and $1.68 \%$ are obtained for $\mathcal{C}^{0}$ linear functions whereas $\mathcal{C}^{2}$ cubic functions give $1.62 \%, 1.46 \%$ and $1.51 \%$. In this case it appears that 32-pixel elements with $\mathcal{C}^{2}$ cubic NURBS is a good compromise between noise sensitivity reduction and discretization error. Figure ?? shows a $3 \mathrm{D}$ visualization of $E_{x x}$ for 16-pixel elements with $\mathcal{C}^{0}$ linear interpolation and for 32-pixel elements with $\mathcal{C}^{2}$ cubic interpolation. Finally Figure ?? presents the evolution of the components $E_{x x}$ (top) and $E_{y y}$ (bottom) of the strain tensor using 32 pixel elements and $\mathcal{C}^{2}$ cubic NURBS at various number of cycles.

\section{Conclusion}

High continuity properties of NURBS functions were exploited in the present paper to improve the performance of strain field measurement by digital image correlation. After a brief reminder of the problem formulation of finite element based DIC, and the definition of the functions used in NURBS based isogeometric analysis, we proposed a new methodology for robust and efficient strain measurement from digital images.

An a priori performance study was performed on an artificially deformed image. After studying the noise sensitivity of the proposed approach, we showed that it allows us to obtain a good balance between interpolation and correlation errors. Indeed we showed that NURBS functions allow us to obtain higher order functions with high continuity with a very small increase in the number of degrees of freedom. When compared to $\mathcal{C}^{0}$ high order polynomial, smooth NURBS produce few functions with a larger support. This results in improving the conditioning of the problem, and consequently reducing noise level and uncertainty. This was demonstrated on an artificially deformed image with a imposed displacement profile specifically designed to test the robustness of the method.

Finally, we applied the proposed method to an experimental example, in which localized strain patterns 
are observed. We compared standard low order finite element DIC techniques with the proposed method and showed its good behavior.

\section{Acknowledgements}

The authors would like to thank Alcan for giving the opportunity to use this numerical approach to an industrial material. Alcan's support is in line with ELiSE research project about thermal exchangers fatigue behavior, which is financed by ANR (ANR-07-MAPR-028) and led jointly by Alcan and MATEIS 36 laboratory. 


\section{References}

[1] M. Sutton, W. Wolters, W. Peters, W. Ranson, S. McNeill, Determination of displacements using an improved digital correlation method, Image Vision Computing 1 (3) (1983) 133-139.

[2] S. Avril, P. Feissel, F. Pierron, F. Villon, Estimation of the strain field from full-field displacement noisy data. Comparing finite elements global least squares and polynomial diffuse approximation, European Journal of Computational Mechanics $17(5-7)(2008) 857-868$.

[3] G. Farin, NURBS Curves and Surfaces: from Projective Geometry to Practical Use, A.K. Peters Ltd., Natick, MA, 1995.

[4] L. Piegl, W. Tiller, The NURBS Book (Monographs in Visual Communication), Springer-Verlag, New York, 2nd edn., 1997.

[5] E. Cohen, R. Riesenfeld, G. Elber, Geometric Modeling with Splines: An Introduction, A.K. Peters Ltd., Wellesley, Massachusetts, 2001.

[6] D. Rogers, An Introduction to NURBS With Historical Perspective, Academic Press, San Diego, CA, 2001.

[7] T. Hughes, J. Cottrell, Y. Bazilevs, Isogeometric analysis: CAD, finite elements, NURBS, exact geometry and mesh refinement, Computer Methods in Applied Mechanics and Engineering 194 (2005) 4135-4195.

[8] J. Cottrell, T. Hughes, Y. Bazilevs, Isogeometric Analysis: Toward Integration of CAD and FEA, Wiley, 2009.

[9] Y. Bazilevs, V. Calo, J. Cottrell, T. Hughes, A. Reali, G. Scovazzi, Variational multiscale residual-based turbulence modeling for large eddy simulation of incompressible flows., Computer Methods in Applied Mechanics and Engineering 197 (1-4) (2007) 173-201.

[10] Y. Bazilevs, V. Calo, T. Hughes, Y. Zhang, Isogeometric fluid-structure interaction: theory, algorithms and computations, Computational Mechanics 43 (1) (2008) 3-37.

[11] I. Akkerman, Y. Bazilevs, V. Calo, T. Hughes, S. Hulshoff, The role of continuity in residual-based variational multiscale modeling of turbulence, Computational Mechanics 41 (3) (2008) 371-378.

[12] H. Gomez, V. Calo, Y. Bazilevs, T. Hughes, Isogeometric analysis of the Cahn-Hilliard phase field model, Computer Methods in Applied Mechanics and Engineering 197 (2008) 4333-4352.

[13] F. Auricchio, L. B. da Veiga, A. Buffa, C. Lovadina, A. Reali, G. Sangalli, A fully locking-free isogeometric approach for plane linear elasticity problems: a stream function formulation, Computer Methods in Applied Mechanics and Engineering 197 (2007) 160-172.

[14] T. Elguedj, Y. Bazilevs, V. Calo, T. Hughes, B-bar and F-bar projection methods for nearly incompressible linear and non-linear elasticity and plasticity based on higher-order NURBS elements, Computer Methods in Applied Mechanics and Engineering 197 (2008) 2732-2762.

[15] T. Hughes, A. Reali, G. Sangalli, Duality and unified analysis of discrete approximations in structural dynamics and wave propagation: Comparison of p-method finite elements with k-method NURBS, Computer Methods in Applied Mechanics and Engineering 197 (49-50) (2008) 4104-4124.

[16] G. Besnard, F. Hild, S. Roux, 'Finite-element' displacement fields analysis from digital images: Application to Portevin-Le Châtelier bands, Experimental Mechanics 46 (6) (2006) 789-803.

[17] M. Sutton, M. Cheng, W. Peters, Y. Chao, S. McNeill, Application of an optimized digital image correlation method to planar deformation analysis, Image Vision Computing 4 (3) (1986) 143-150.

[18] M. Sutton, S. McNeill, J. Helm, Y. Chao, Photomechanics, chap. Advances in two-dimensional and three-dimensional computer vision, Springer, 323-372, 2000.

[19] P. Cheng, M. A. Sutton, H. W. Schreier, S. R. McNeill, Full-field Speckle Pattern Image Correlation with B-Spline Deformation Function, Experimental Mechanics 42 (3) (2002) 344-352.

[20] M. Bornert, F. Brémand, P. Doumalin, J. Dupré, M. Fazzini, M. Grédiac, F. Hild, S. Mistou, J. Molimard, J. Orteu, 
L. Robert, Y. Surrel, P. Vacher, B. Wattrisse, Assessment of Digital Image Correlation Measurment Errors: Methodology and Results, Experimental Mechanics 49 (3) (2009) 353-370.

431 [21] J. Réthoré, F. Hild, S. Roux, Shear-band capturing using a multiscale extended digital image correlation technique, Computer Methods in Applied Mechanics and Engineering 196 (49-52) (2007) 5016-5030.

[22] J. Réthoré, G. Besnard, G. Vivier, F. Hild, S. Roux, Experimental investigation of localized phenomena using Digital Image Correlation, Philosophical Magazine 88 (28-29) (2008) 3339-3355. 


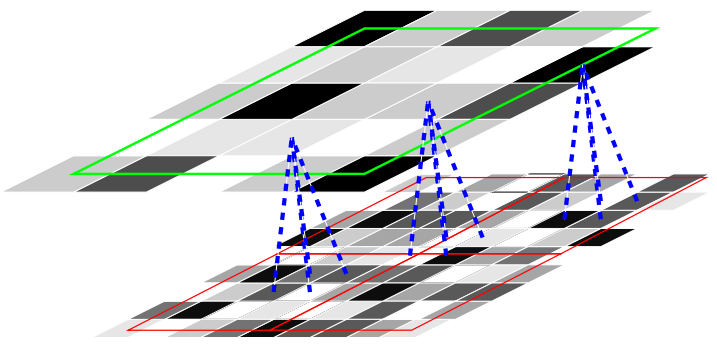

(a)

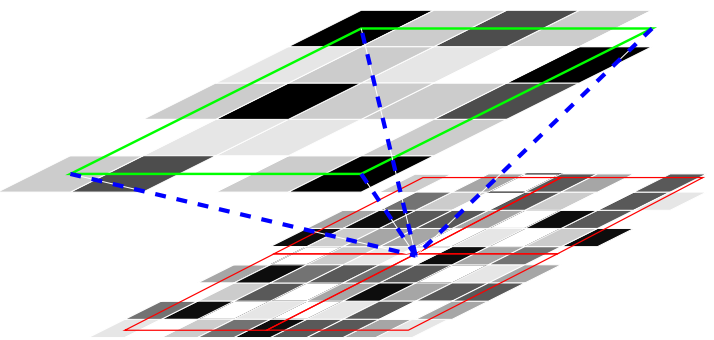

(b)

Figure 1: Illustration of the restriction operation (left) and the prolongation operation (right).

Zone of Interest and mesh defined on the digital image

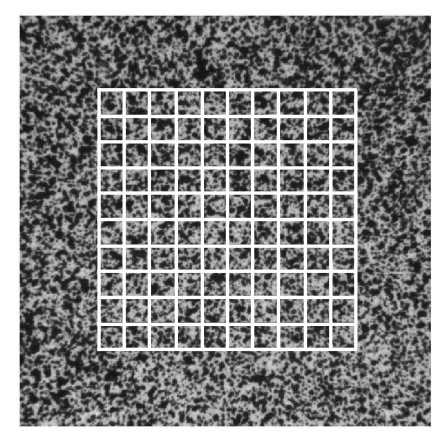

Mesh extraction and knot vector definition based on pixel numbering

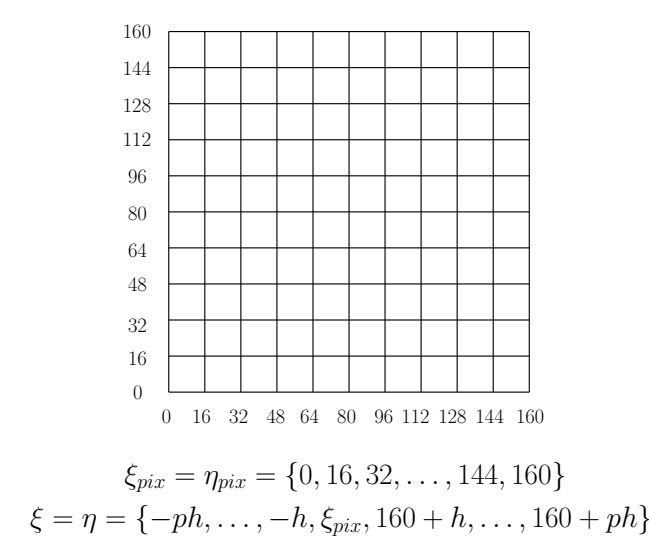

Basis functions construction from knot vectors

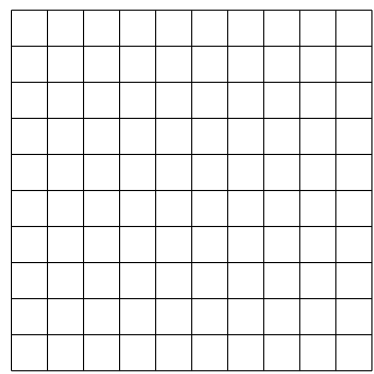

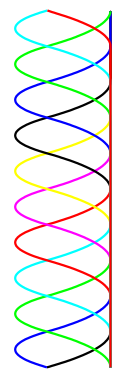
AXX⿻从人

Figure 2: Zone of interest and mesh definition on digital image (left), computational mesh extraction and knot vectors creation from pixel numbering (center), and basis functions evaluation from knot vectors (right).

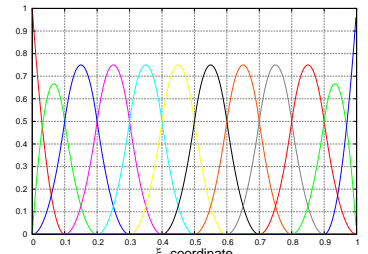

(a)

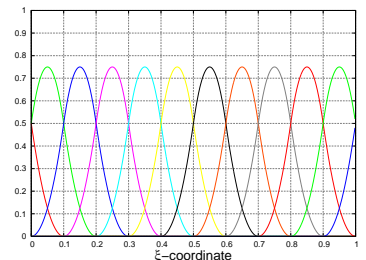

(e)

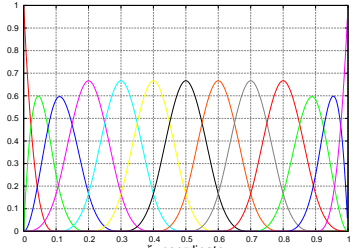

(b)

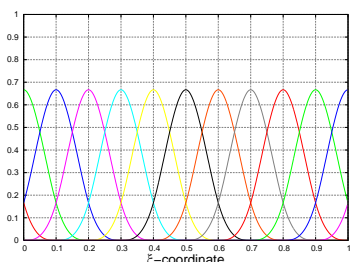

(f)

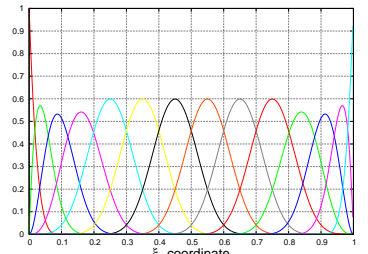

(c)

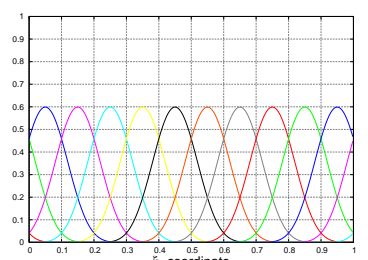

$(\mathrm{g})$

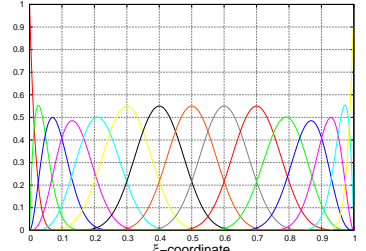

(d)

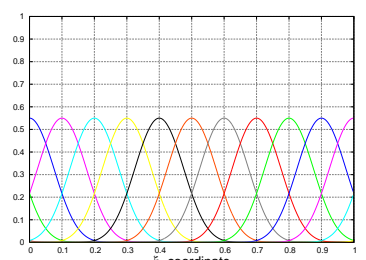

(h)

Figure 3: Unidimensional B-Spline basis functions with $p=2$ (left) to $p=5$ (right) with open knot vectors (top) and periodic knot vector (bottom) 


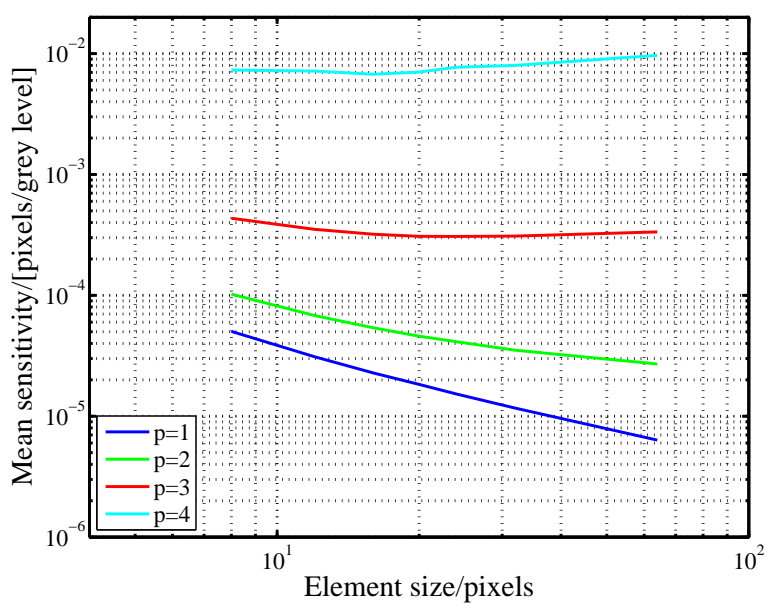

(a)

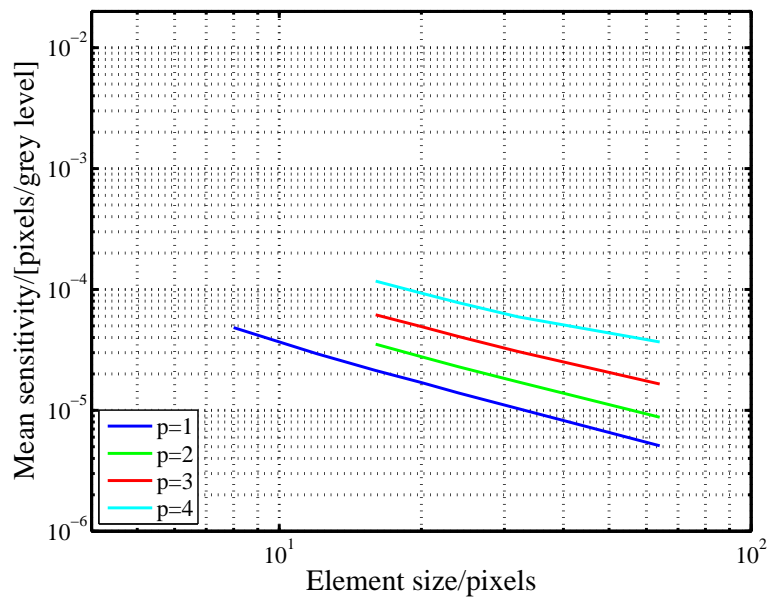

(c)

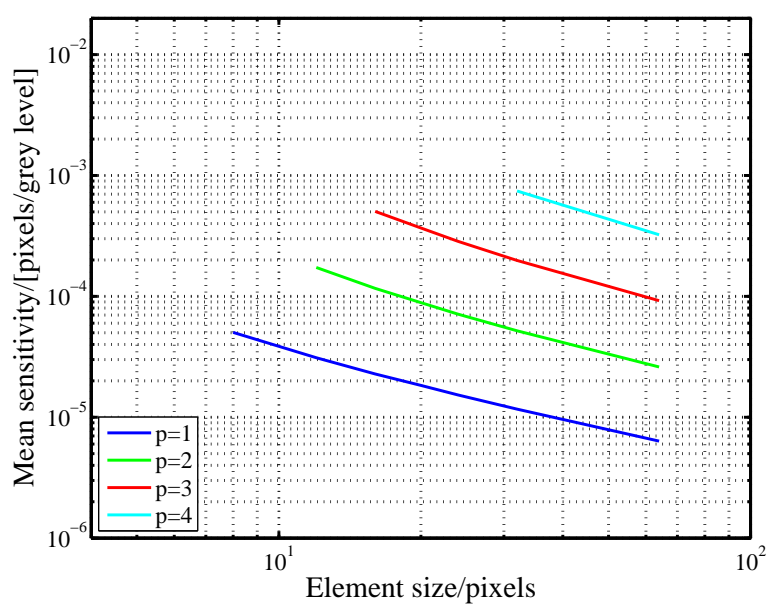

(b)

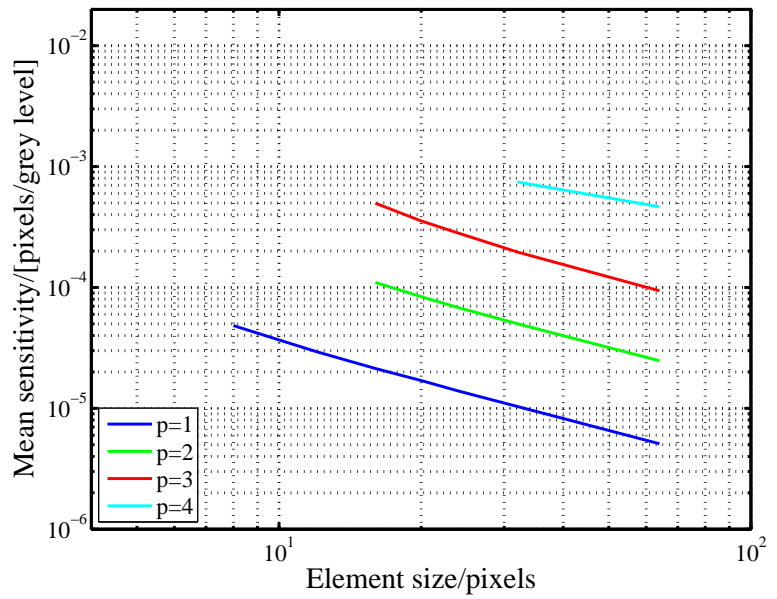

(d)

Figure 4: Mean sensitivity in pixels/gray levels versus element size for (a) $\mathcal{C}^{p-1}$-continuous NURBS and (b) $\mathcal{C}^{0}$-continuous NURBS, and without the degrees of freedom that have support on the boundary elements for (c) $\mathcal{C}^{p-1}$-continuous NURBS and (d) $\mathcal{C}^{0}$-continuous NURBS. 

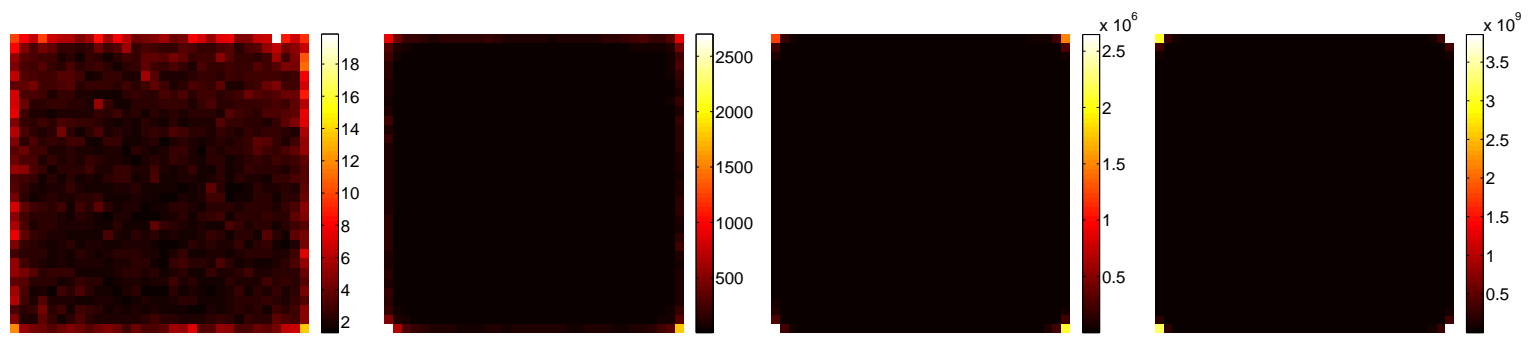

(a)
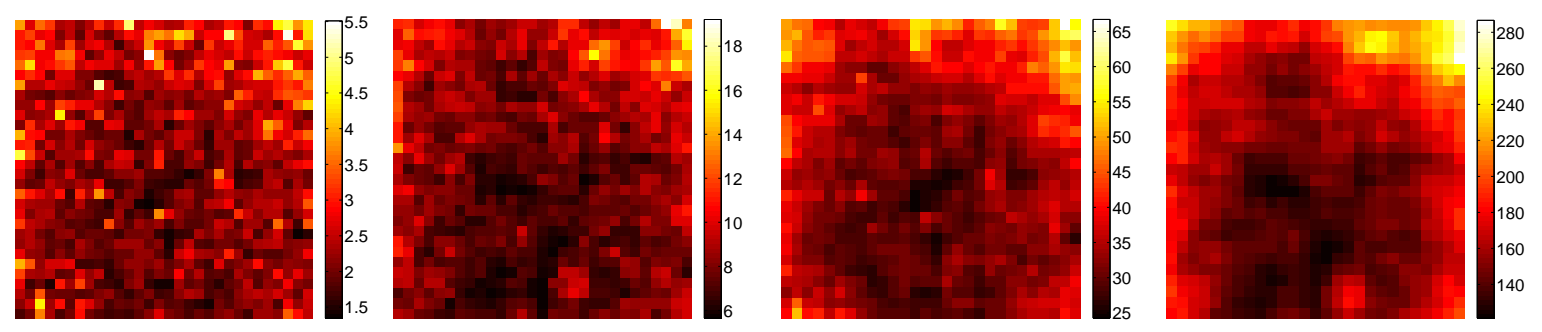

(b)

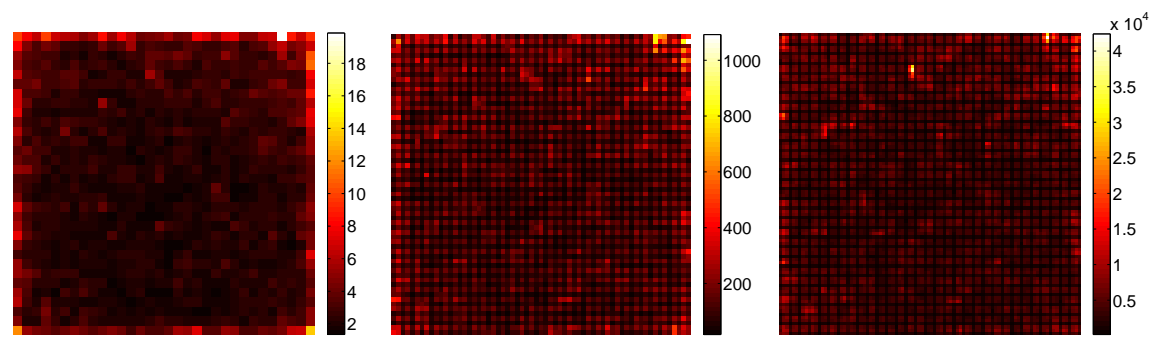

(c)
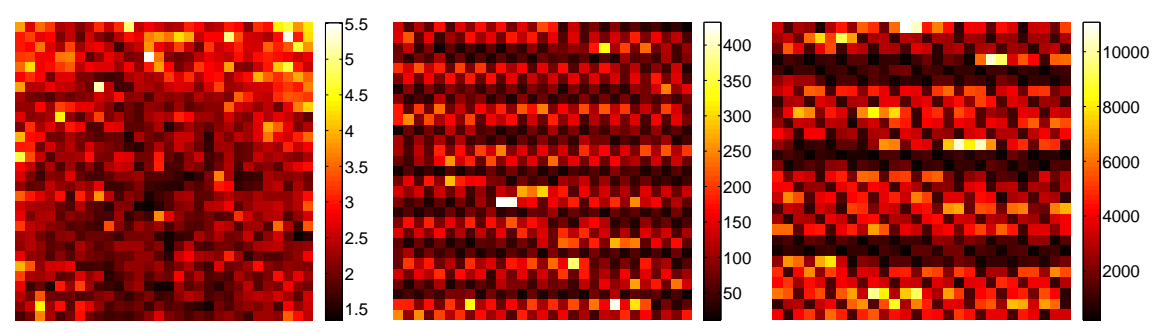

(d)

Figure 5: Sensitivity map in pixel of the degrees of freedom in the vertical direction. (a) $\mathcal{C}^{p-1}$ NURBS with 16 pixels per elements for $p=1 \ldots 4$ (left to right). (b) $\mathcal{C}^{p-1}$ NURBS with 16 pixels per elements for $p=1 \ldots 4$ without the degrees of freedom that have support on the boundary elements. (c) $\mathcal{C}^{0}$ NURBS with 16 pixels per elements for $p=1 \ldots 3$. (d) $\mathcal{C}^{0}$ NURBS with 16 pixels per elements for $p=1 \ldots 3$ without the degrees of freedom that have support on the boundary elements. 


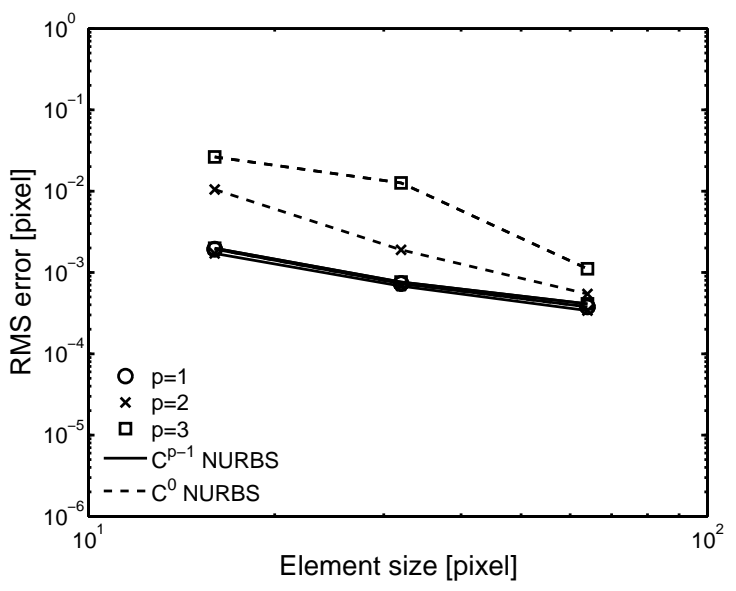

(a)

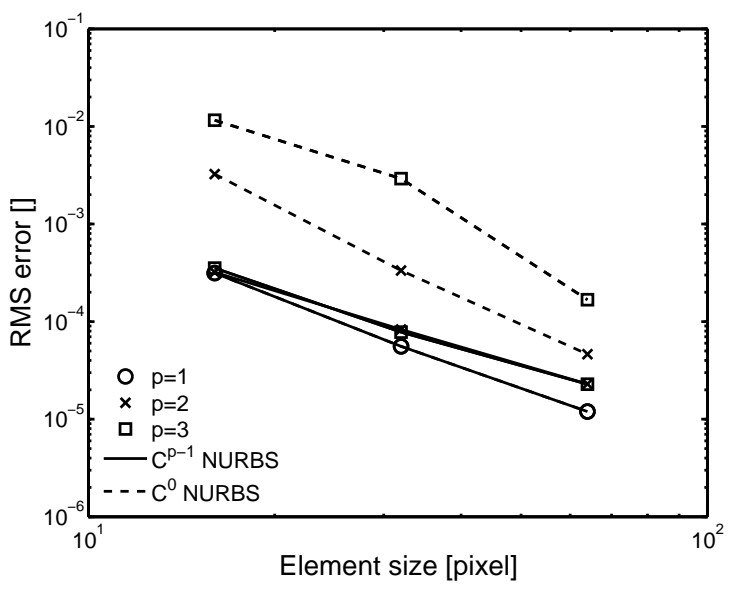

(c)

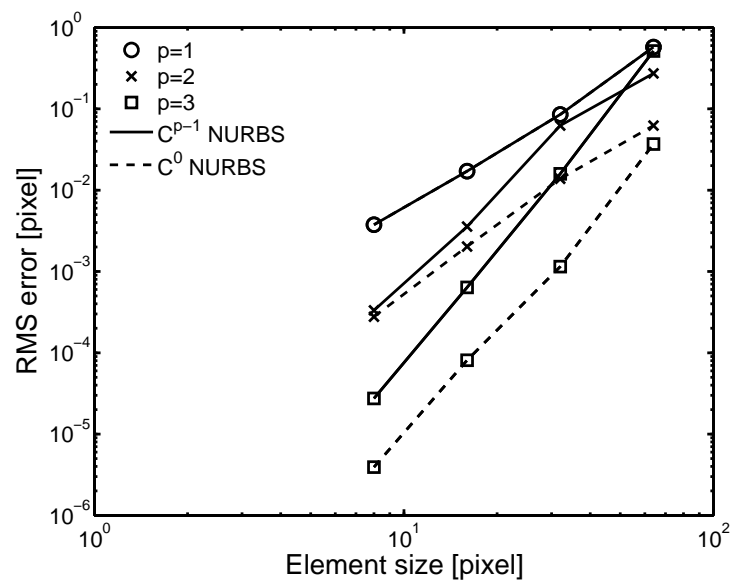

(b)

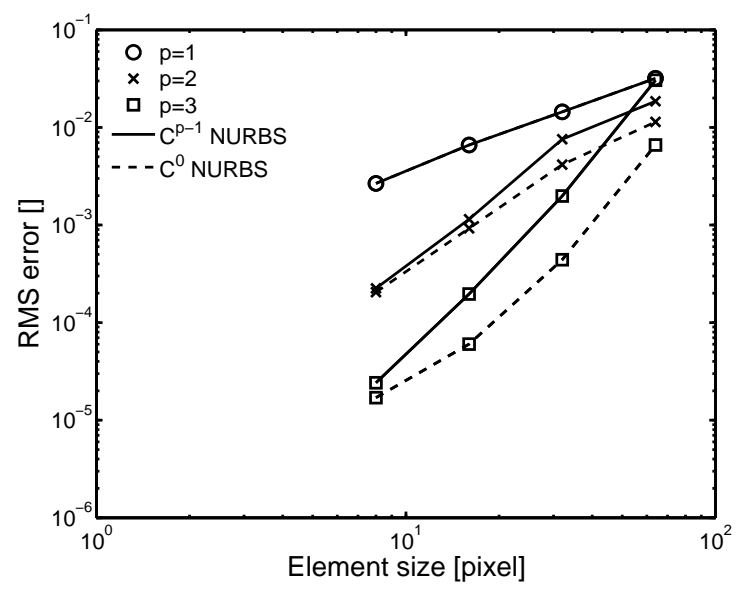

(d)

Figure 6: Evolution of the correlation error (left) and interpolation error (right) as a function of the element size for the displacement (top) and strain (bottom). 


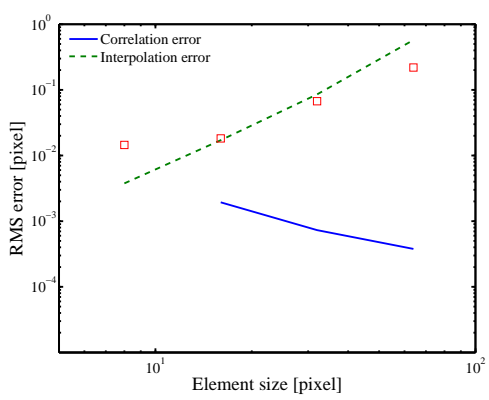

(a)

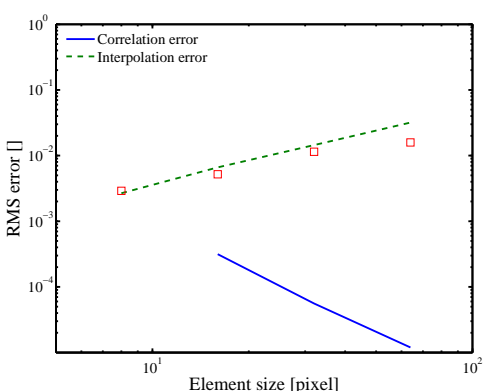

(d)

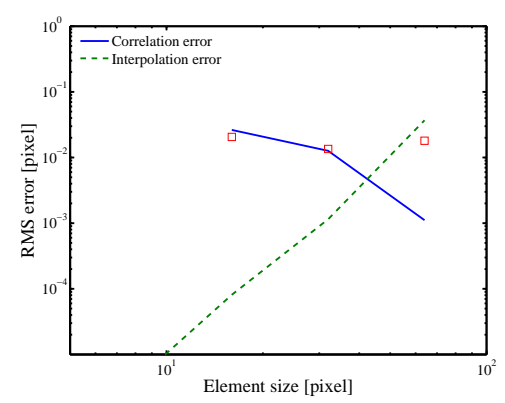

(b)

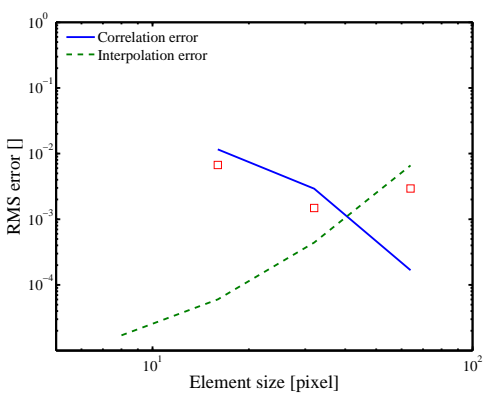

(e)

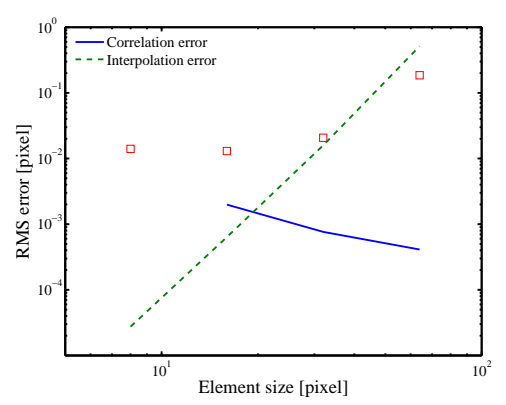

(c)

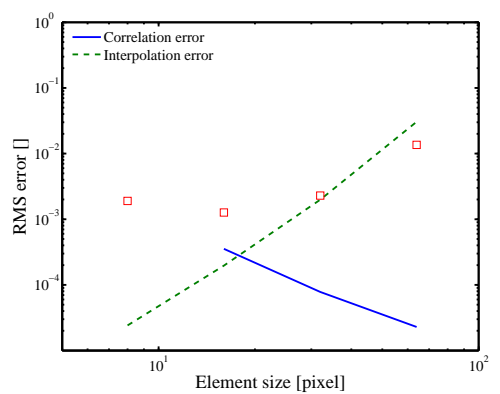

(f)

Figure 7: Measurement uncertainty on displacement (top) and strain (bottom). From left to right $\mathcal{C}^{0}$ piecewise linear, $\mathcal{C}^{0}$ piecewise cubic and $\mathcal{C}^{2}$ piecewise cubic NURBS. The total error is depicted with square marks, the correlation error with lines and the interpolation error with dashed lines. 

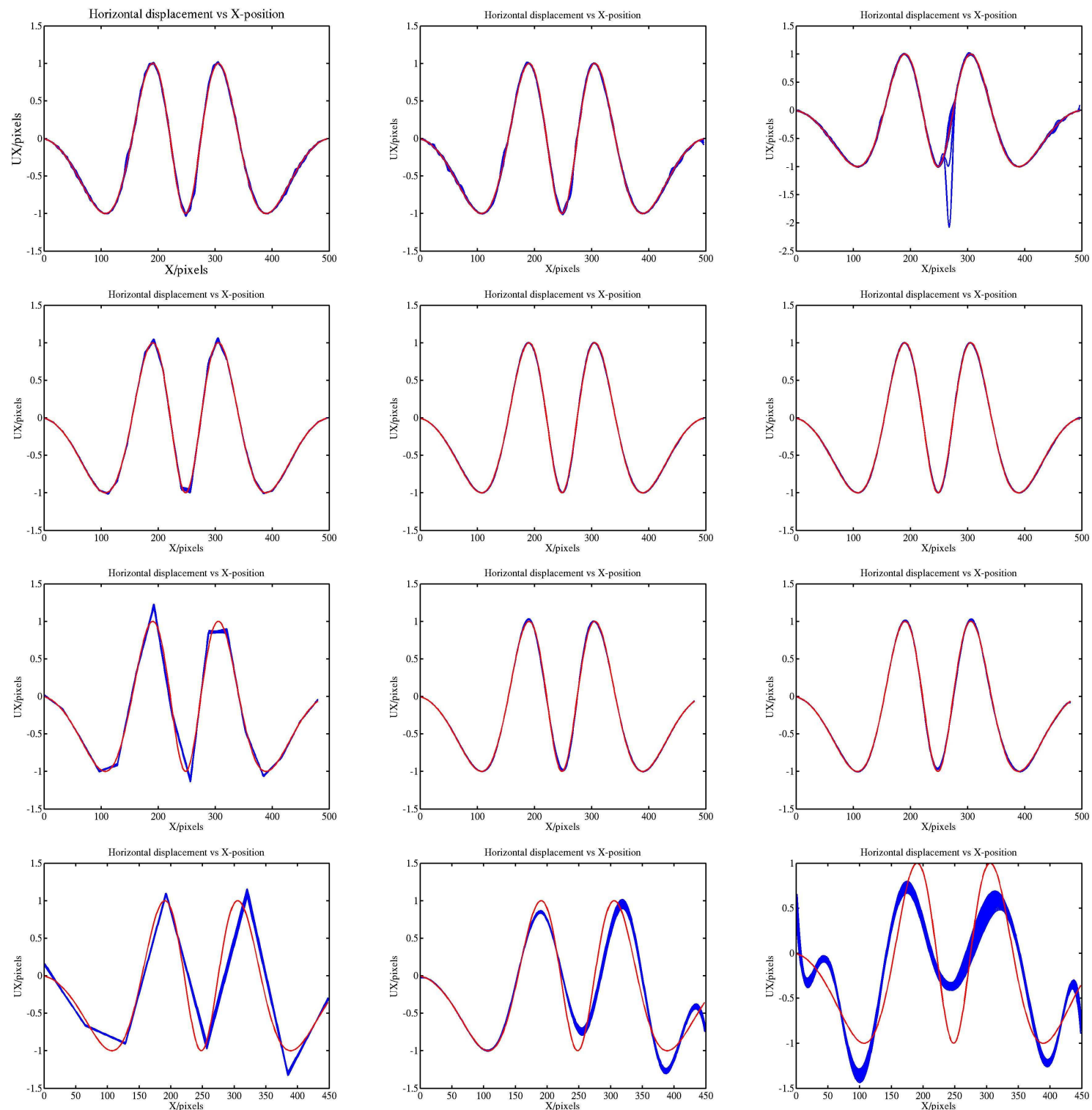

Figure 8: Artificial test. Imposed and computed horizontal displacement profiles for $\mathcal{C}^{p-1}$-continuous $k$-refined NURBS with 8, 16, 32 and 64 pixels per element for $p=1$ (left), $p=3$ (center) and $p=4$ (right). 

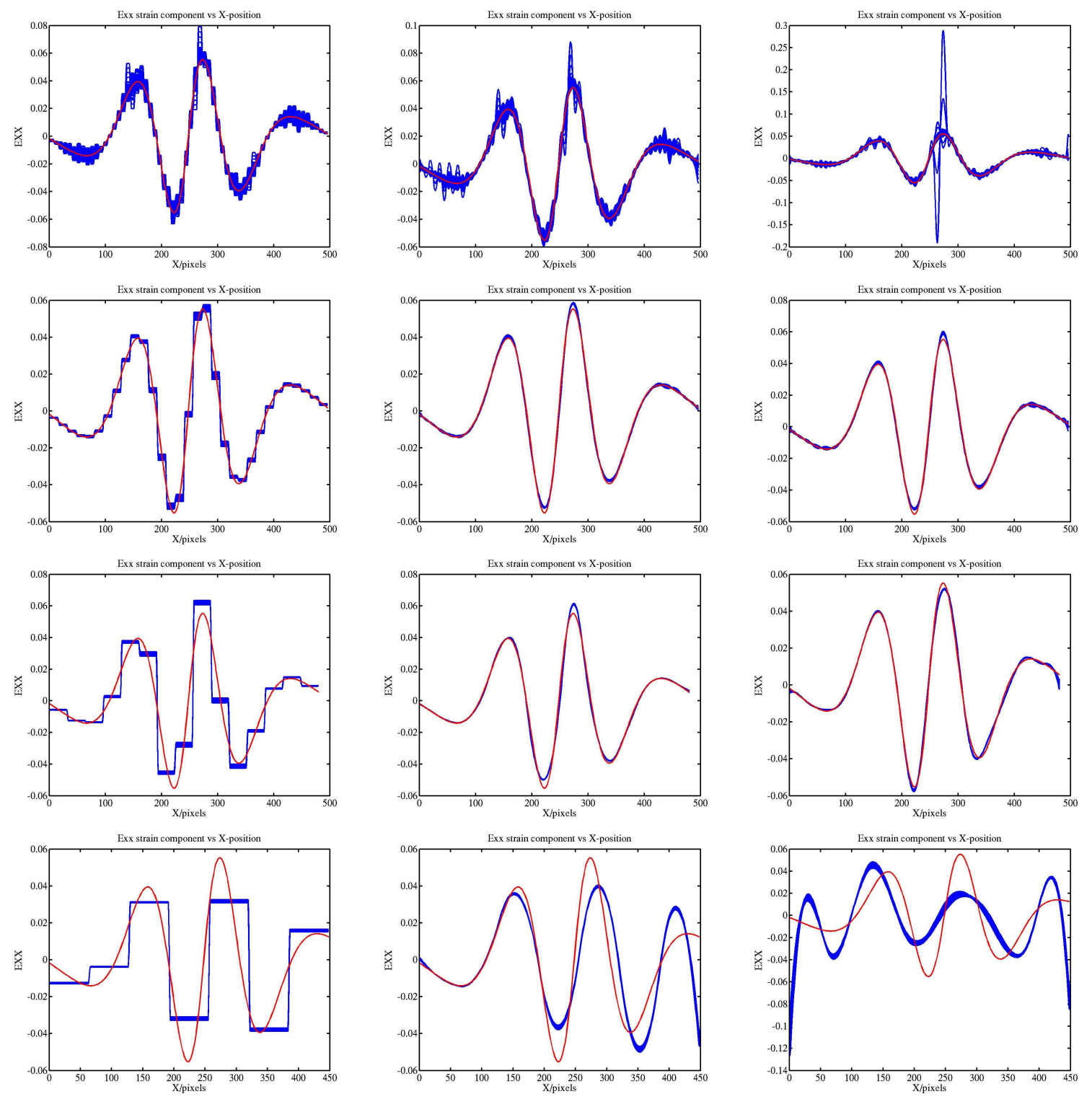

Figure 9: Artificial test. Imposed and computed $\varepsilon_{x x}$ strain component profiles for $\mathcal{C}^{p-1}$-continuous $k$-refined NURBS with 8 , 16, 32 and 64 pixels per element for $p=1$ (left), $p=3$ (center) and $p=4$ (right). 

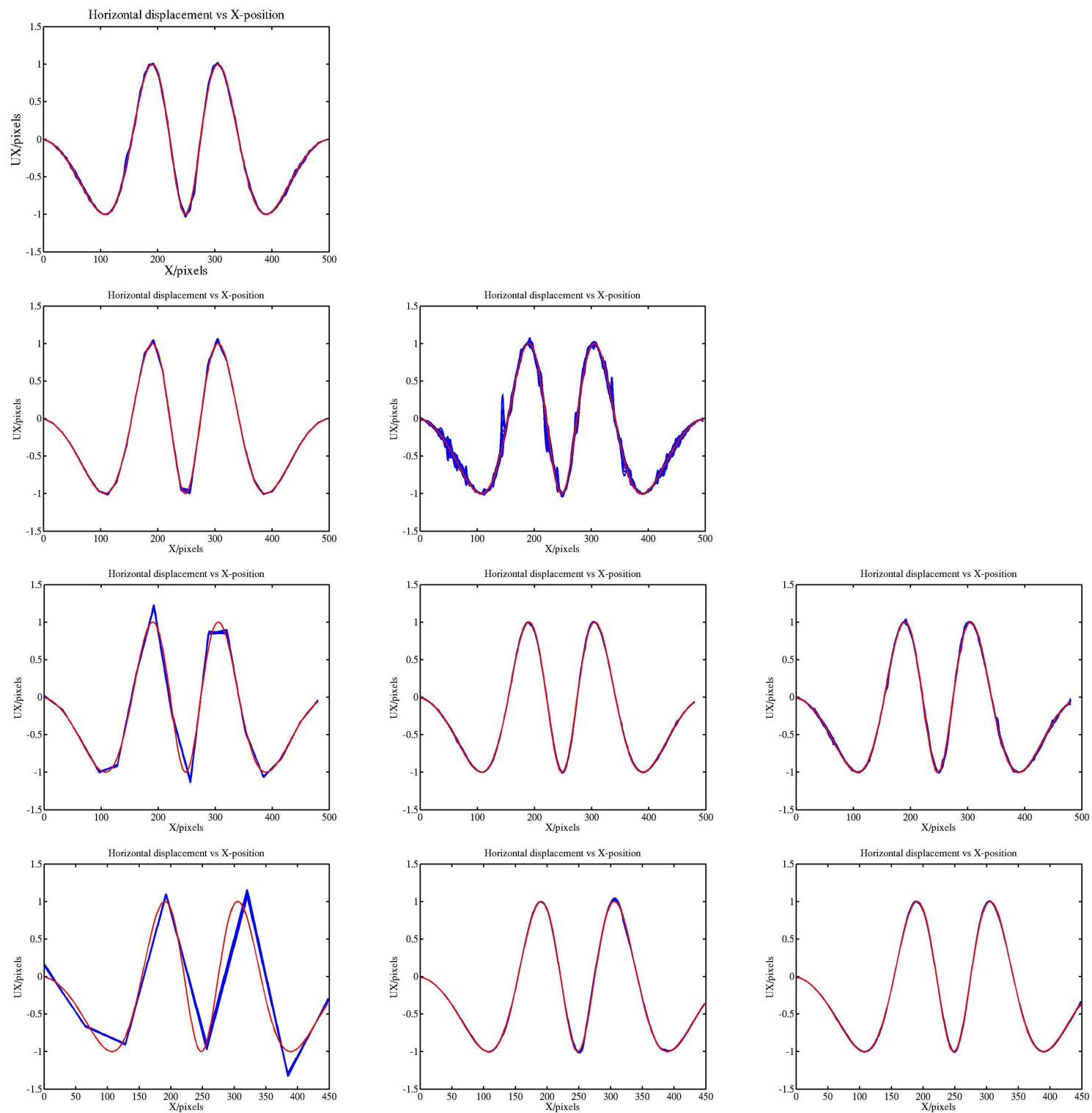

Figure 10: Artificial test. Imposed and computed horizontal displacement profiles for $\mathcal{C}^{0}$-continuous NURBS with $8,16,32$ and 64 pixels per element for $p=1$ (left), $p=3$ (center) and $p=4$ (right). 

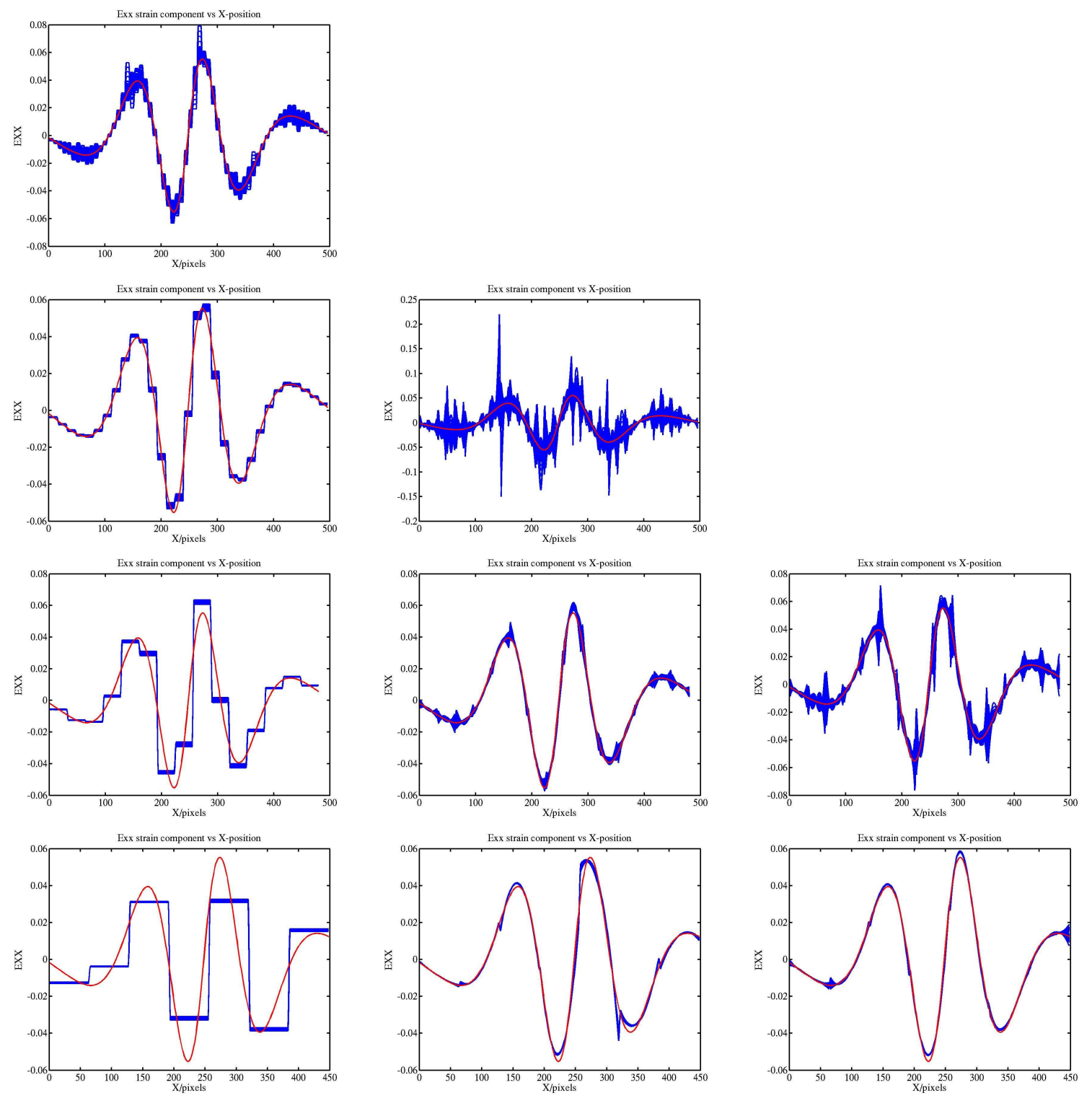

Figure 11: Artificial test. Imposed and computed $\varepsilon_{x x}$ strain component profiles for $\mathcal{C}^{0}$-continuous NURBS with $8,16,32$ and 64 pixels per element for $p=1$ (left), $p=3$ (center) and $p=4$ (right). 


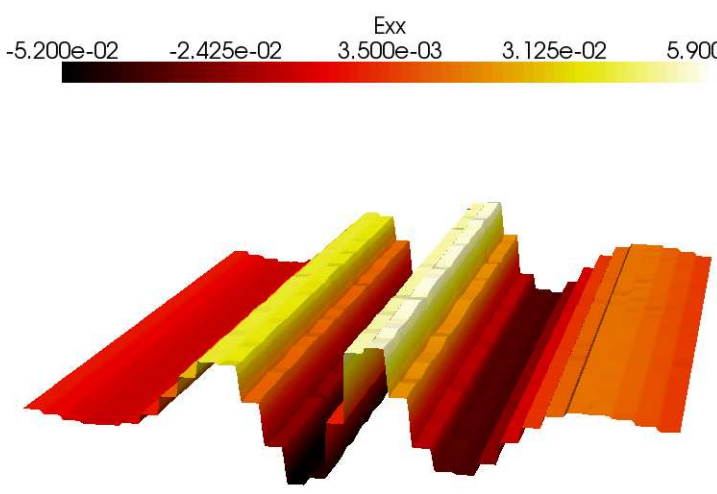

(a)

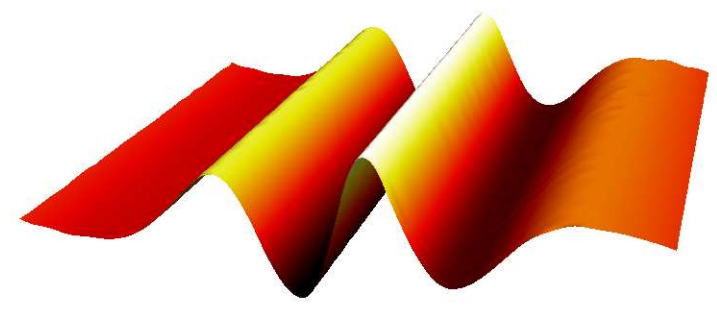

(c)

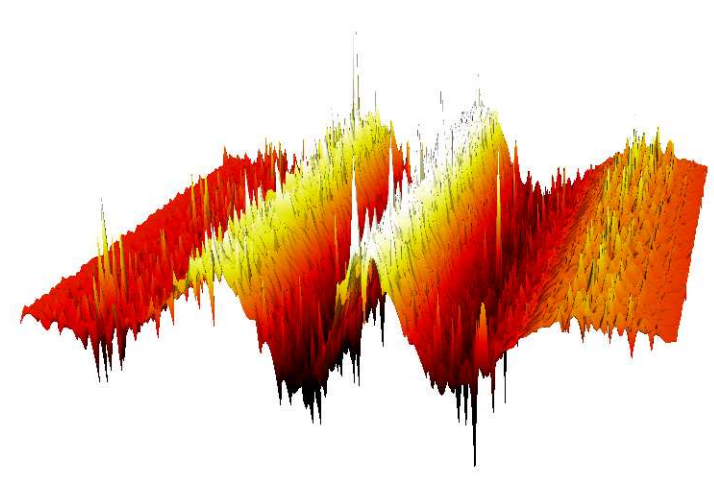

(e)

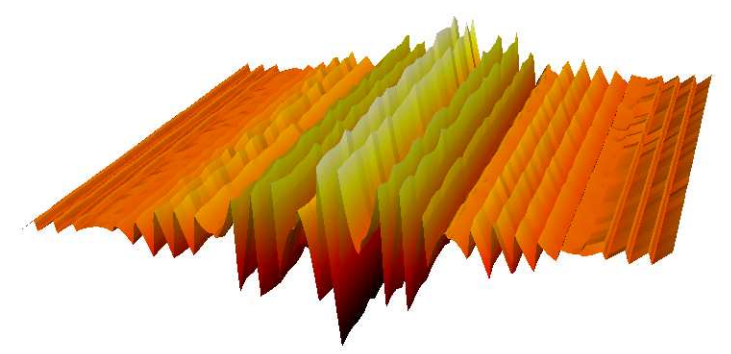

(b)

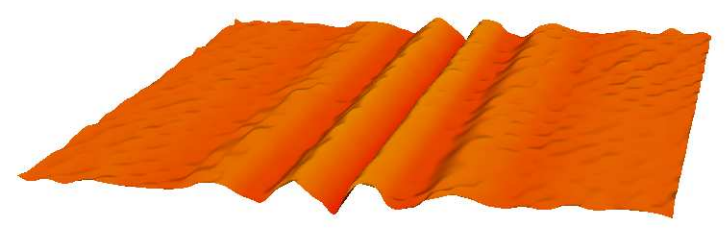

(d)

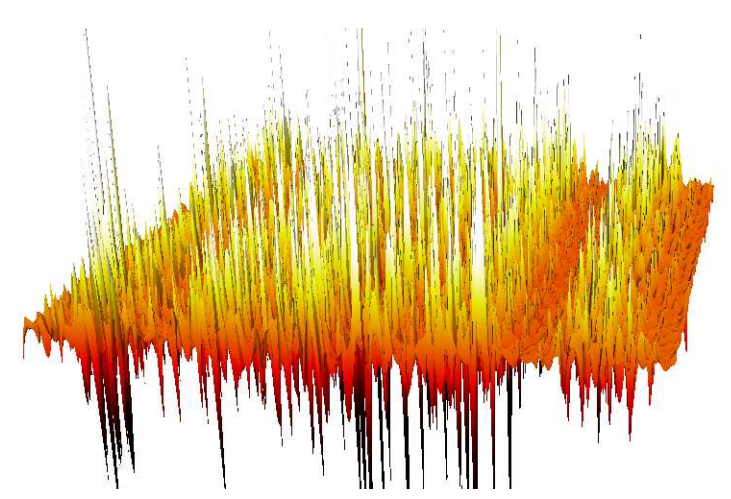

(f)

Figure 12: Artificial test: 3D visualisation of the $\varepsilon_{x x}$ strain component (on the left, amplified 1000 times), and the error on the $\varepsilon_{x x}$ strain component (on the right, amplified 3000 times) for a mesh of 16 -pixels per elements with $\mathcal{C}^{0}$ linear (top), $\mathcal{C}^{2}$ cubic (middle) and $\mathcal{C}^{0}$ cubic (bottom) NURBS. 


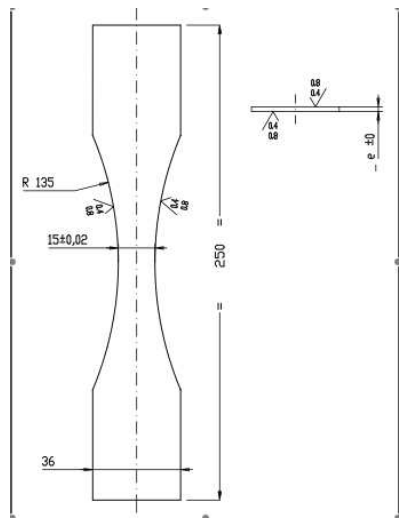

(a)

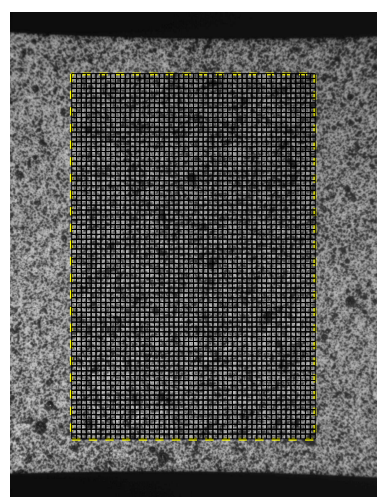

0 cycle

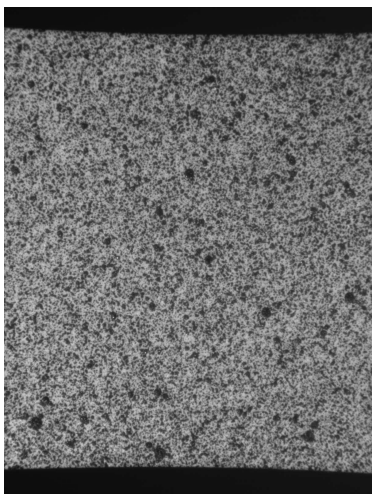

6000 cycles

(c)

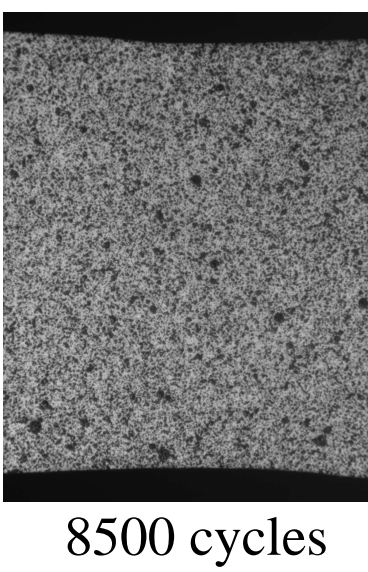

(d)

Figure 13: (a): sample geometry. (b): reference image $(1200 \times 1600$ pixels with 8-bit digitization $)$ domain of interest and mesh for 16-pixel elements. (c): deformed image after 6000 cycles. (d): deformed image after 8500 cycles. 


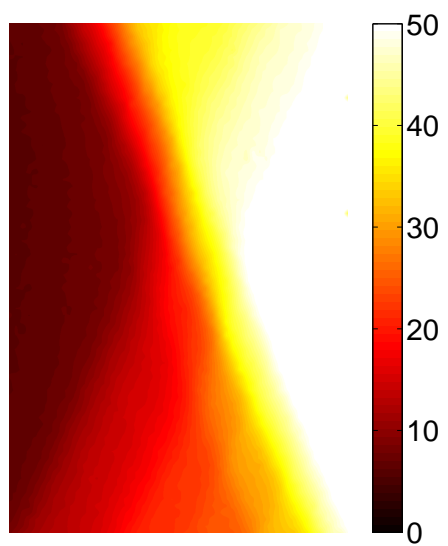

(a)

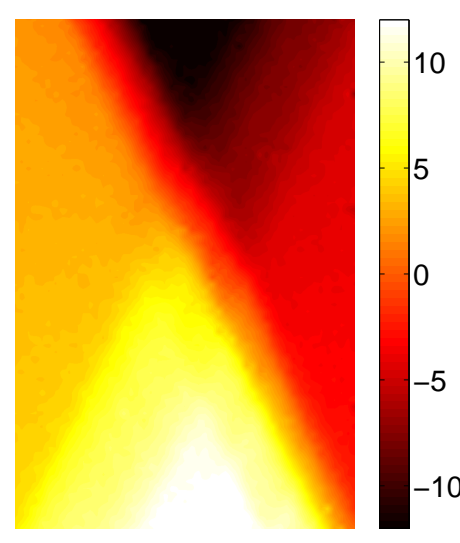

(b)

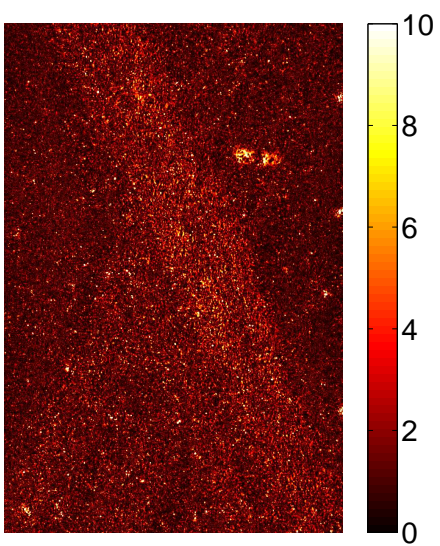

(c)

\section{Deformed mesh (x5)}
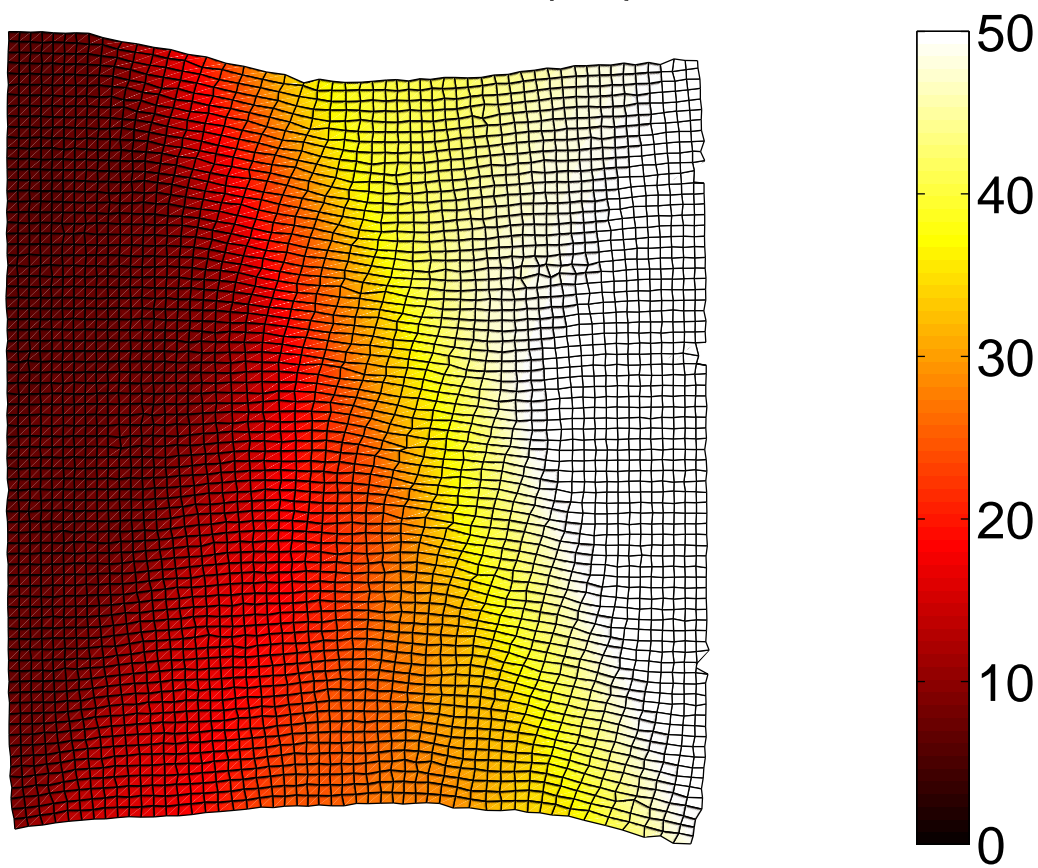

(d)

Figure 14: For 16-pixel elements with $\mathcal{C}^{0}$ piecewise linear functions: horizontal (a) and vertical (b) displacement after 8500 cycles in pixel. (c) shows the local correlation error in \% of the dynamic of the reference image (223 gray levels) and (d) the deformed mesh. 


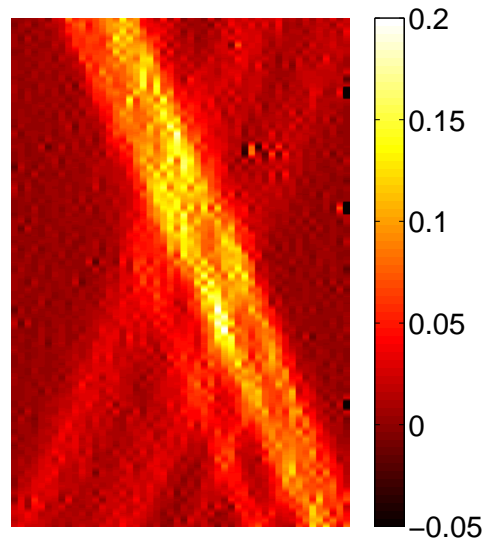

(a)

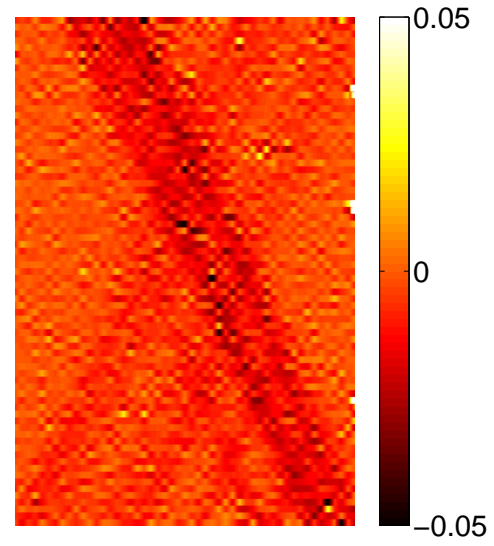

(b)

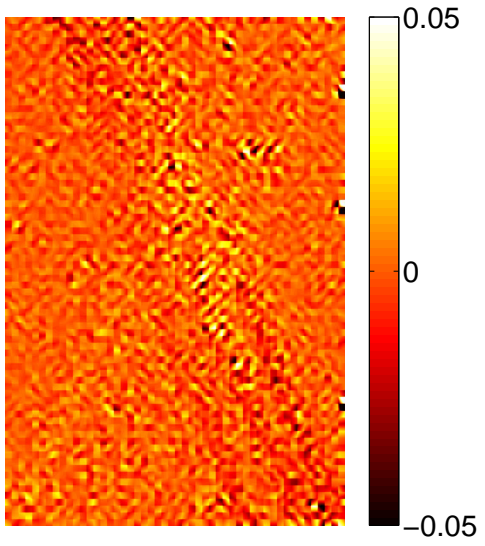

(c)

Figure 15: For 16-pixel elements with $\mathcal{C}^{0}$ piecewise linear functions: $E_{x x}$ (a), $E_{y y}(\mathrm{~b})$ and $E_{x y}$ (c) components of the GreenLagrange strain tensor after 8500 cycles. 


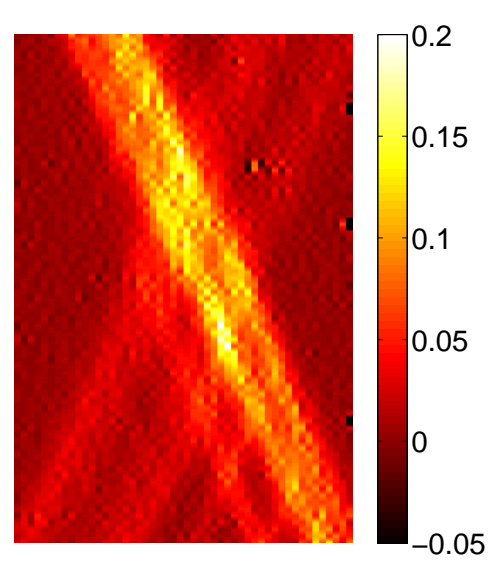

(a)

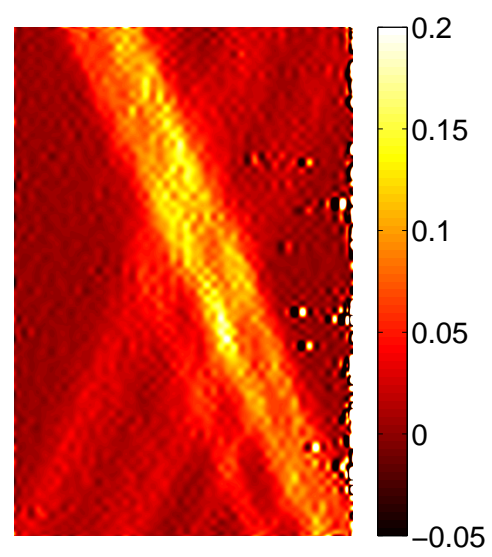

(d)

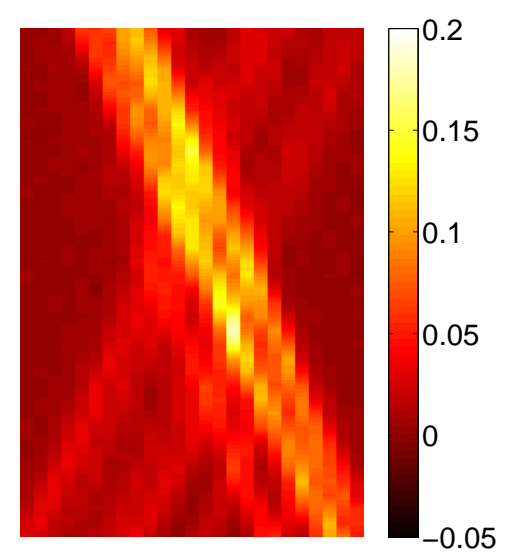

(b)

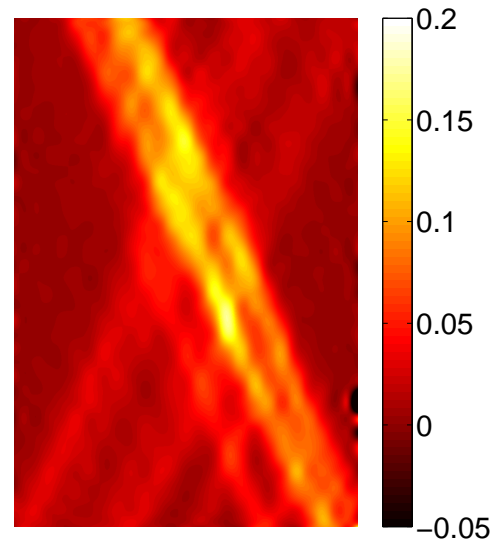

(e)

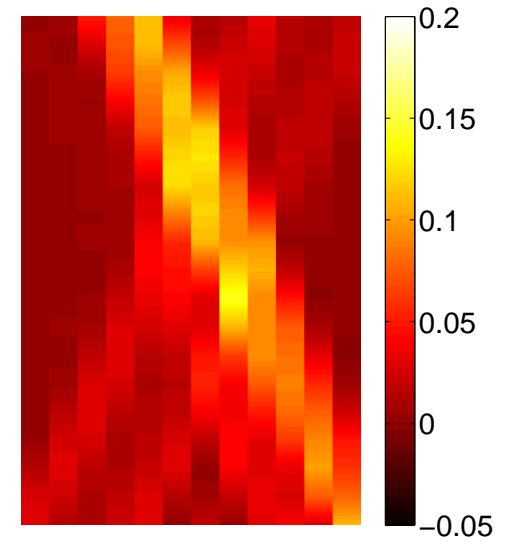

(c)

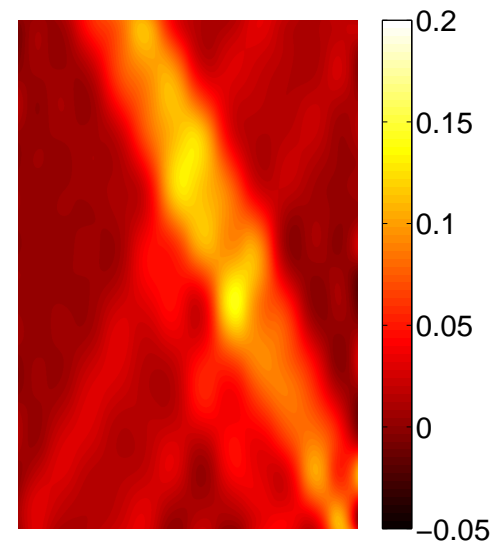

(f)

Figure 16: Comparison of $E_{x x}$ for different mesh size and interpolation degrees after 8500 cycles: from left to right 16, 32 and 64-pixel elements, top $\mathcal{C}^{0}$ linear functions, bottom $\mathcal{C}^{2}$ cubic functions. 


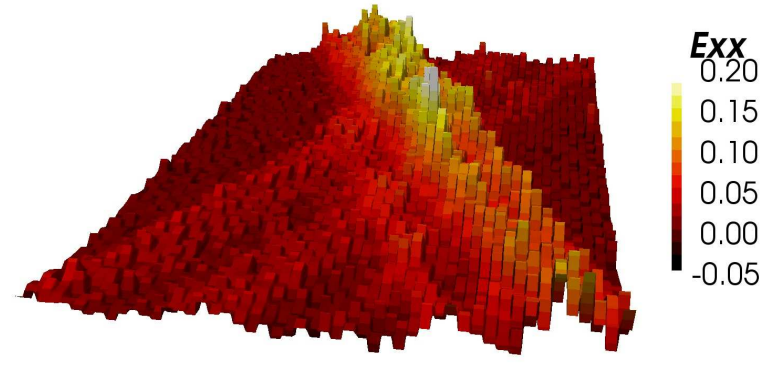

(a)

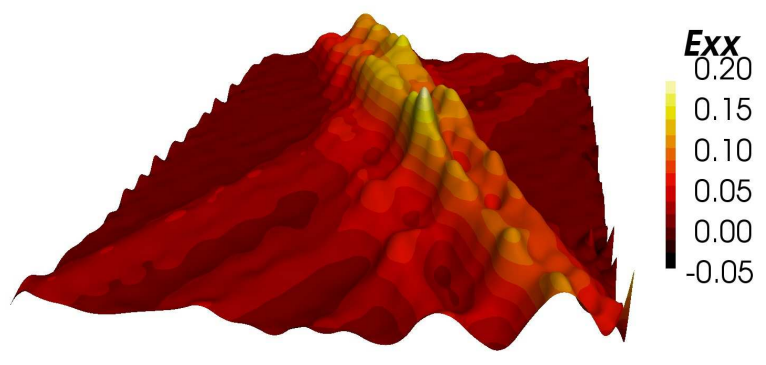

(b)

Figure 17: 3D visualization of $E_{x x}$ after 8500 cycles for 16-pixel elements with $\mathcal{C}^{0}$ linear functions (left) and for 32-pixel elements with $\mathcal{C}^{2}$ cubic functions (right). 


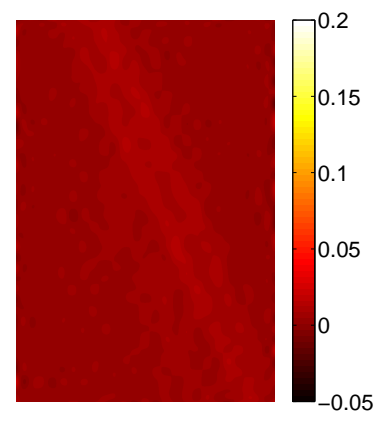

(a)

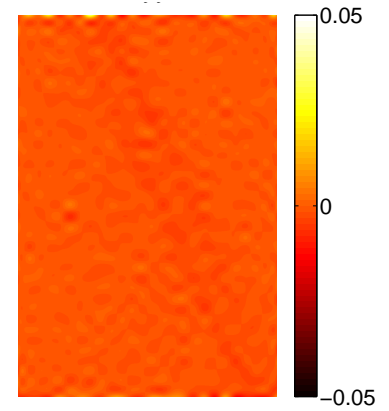

(e)

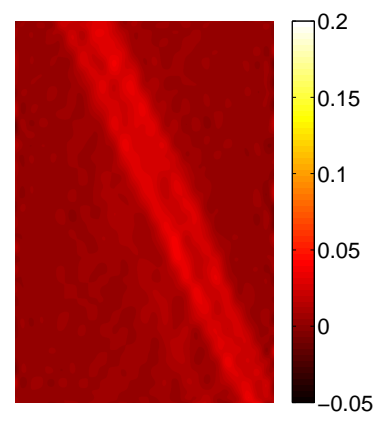

(b)

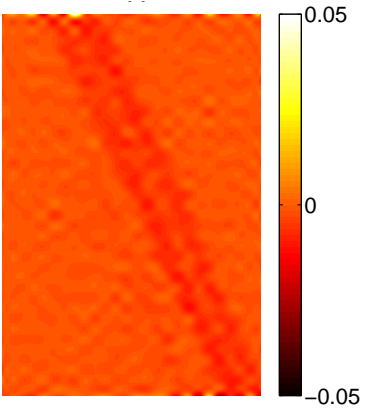

(f)

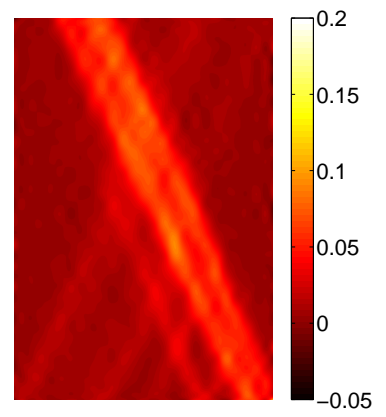

(c)

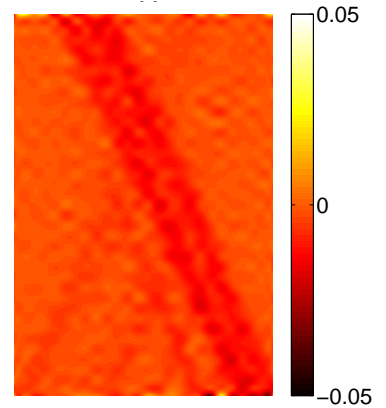

(g)

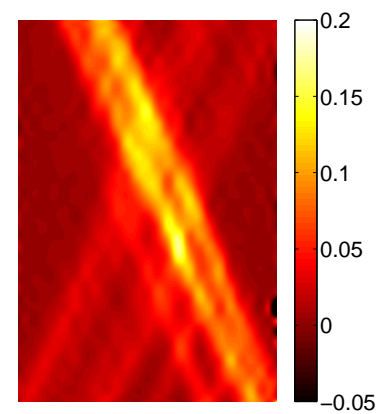

(d)

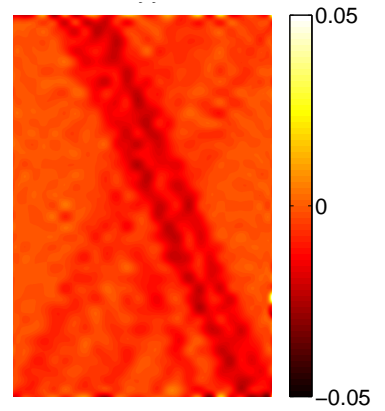

(h)

Figure 18: Evolution of $E_{x x}$ (top) and $E_{y y}$ (bottom) for 32-pixel elements with $\mathcal{C}^{2}$ cubic functions: (a,e) 6000 cycles, (b,f) 7000 cycles, $(\mathrm{c}, \mathrm{g}) 8000$ cycles and $(\mathrm{d}, \mathrm{h}) 8500$ cycles. 